\title{
19. OXYGEN AND CARBON ISOTOPE STRATIGRAPHY AT SITE 723, OMAN MARGIN1
}

\author{
Nobuaki Niitsuma, ${ }^{2}$ Tadamichi Oba, ${ }^{3}$ and Makoto $\mathrm{Okada}^{4}$
}

\begin{abstract}
Site 723 is located in a water depth of $808 \mathrm{~m}$ at the center of the oxygen minimum zone and the middle part of the main thermocline on the Oman Margin.

Oxygen isotope curves of planktonic $\delta^{18} \mathrm{O}_{\mathrm{P}}$ and benthic $\delta^{18} \mathrm{O}_{\mathrm{B}}$ can be traced back continuously to Stage 23 with high resolution measurements. A tentative correlation to Stage 53 has been tried using oxygen isotope stratigraphy. The amplitudes of the fluctuations of the benthic $\delta^{18} \mathrm{O}_{\mathrm{B}}$ curve are small, compared with the planktonic $\delta^{18} \mathrm{O}_{\mathrm{P}}$ curve. The delays of benthic oxygen isotopes $\delta^{18} \mathrm{O}_{\mathrm{B}}$ related to the planktonic $\delta^{18} \mathrm{O}_{\mathrm{P}}$ appear in the transgressive stages.

Carbon isotopes of benthic $\delta^{13} \mathrm{C}_{\mathrm{B}}$ and planktonic $\delta^{13} \mathrm{C}_{\mathrm{P}}$ generally show an inverse correlation with oxygen isotope values $\delta^{18} \mathrm{O}_{\mathrm{B}}$ and $\delta^{18} \mathrm{O}_{\mathrm{P}}$, however, the changes of $\delta^{13} \mathrm{C}$ are more gradual than those of $\delta^{18} 0$ during transgressive stages in spite of the synchronized changes of $\delta^{13} \mathrm{C}$ with those of $\delta^{18} 0$ during regressive stages.

The difference of oxygen isotope between benthic and planktonic foraminifers represents the degree of pushing up the thermocline by upwelling, and the difference of carbon isotope represents the relative amount of upwelling $\mathrm{\Sigma CO}_{2}$ to the biological uptake in the surface water. These isotopic differences can be used as indicators of upwelling and show strong upwelling in the interglacial and weak upwelling in the glacial stages. The organic carbon content is correlated with the isotopic upwelling indicators, and higher content is correlated with the isotopic upwelling indicators and higher content appears in the interglacial stages.

The calculated rate of sedimentation based on oxygen isotope stratigraphy in glacial stages is significantly high, two to four times that of interglacial stages, and the absolute flux of fluvial sediments with variability of lithofacies increased in the glacial stage.

The present glacial-interglacial cycle with the fluctuation of upwelling relating to the southwest monsoon can be traced back to Stage $8,250 \mathrm{ka}$. From Stage 8 to $12,250-450 \mathrm{ka}$, the upwelling indicator of oxygen isotope difference did not show such distinct cyclicity. For Stages 12-15, 450-600 ka, the upwelling can be estimated as strong as in interglacial stage of the present cycles, with slightly weak upwelling in the glacial stage. This upwelling and climate can be traced back to the late Pliocene. The strongest upwelling can be estimated in the Pliocene-Pleistocene time by the isotopic indicators and the high organic carbon content.
\end{abstract}

\section{INTRODUCTION}

The primary object of ODP Leg 117, Neogene Package, was to clarify the monsoonal upwelling history in the northwestern Indian Ocean. To achieve this goal a series of sites was drilled along a depth transect in the Oman Margin, crossing through the oxygen minimum zone and thermocline (Prell, Niitsuma, et al., 1989; Figs. 1 and 2).

This paper presents the results of high resolution isotopic measurements on the sedimentary sequence of Site 723 located on the center of the oxygen minimum zone and the middle part of the thermocline, and discusses the paleoclimatology and history of the upwelling on the Oman Margin, related to the southwest monsoon in the northwestern Indian Ocean.

\section{SITE}

Site 723 is located in the center of the mid-slope basin of the Oman Margin. The subsiding sedimentary basin developed along the inner side of the sill which consists of tectonically growing enechelon anticlines resulting from right lateral movement (Fig. 1).

\footnotetext{
${ }^{1}$ Prell, W. L., Niitsuma, N., et al., 1991. Proc. ODP, Sci. Results, 117: College Station, TX (Ocean Drilling Program).

${ }^{2}$ Institute of Geosciences, Shizuoka University, Shizuoka, 422 Japan.

3 Department of Geology, College of Liberal Arts, Kanazawa University, Kanazawa, 920 Japan.

4 Ocean Research Institute, University of Tokyo, Tokyo, 164 Japan.
}

\section{SAMPLES}

Above 85 mbsf, Hole 723A was drilled by advanced hydraulic piston corer (APC). However, below 40 mbsf, gas expansion developed after the core recovery. Below $85 \mathrm{mbsf}$, the extended core barrel (XCB) was used and gave poor recovery. Hole 723B was drilled by APC for upper $80 \mathrm{~m}$, and then drilled by XCB. The apparent recovery was almost complete above $140 \mathrm{mbsf}$, however, gas expansion appeared from $40 \mathrm{mbsf}$ and developed seriously below 80 mbsf.

The recovered sediments consist of marly nannofossil ooze intercalated by finely laminated siliceous-marly ooze and dolomitic calcareous concretions.

Shipboard sampling at $75 \mathrm{~cm}$ intervals was done by taking two samples routinely from each section all through the drilled sediments of Hole 723A (0-384.77 mbsf) and 723B (82.00$405.06 \mathrm{mbsf}$ ). Sample volume was $10 \mathrm{~cm}^{3}$. Shore-based sampling at 20-25 cm intervals was done for 0-91.31 mbsf of Hole $723 \mathrm{~A}$ and from 86.66 to $140.18 \mathrm{mbsf}$ of Hole 723B. Furthermore, $20-25 \mathrm{~cm}$ interval samples from 17 to 82 mbsf of Hole $723 \mathrm{~B}$ were taken to complete the missing stratigraphic intervals in Hole $723 \mathrm{~A}$, caused by the coring gaps and whole-round geochemical sampling gaps, based on precise layer-by-layer correlation (Niitsuma, this volume). The sampling gaps from gas expansions in Hole $723 \mathrm{~B}$ at $100,110,120$, and 130 mbsf could not be filled by Hole $723 \mathrm{~A}$ sampling because of poor recovery and lack of lithologic markers.

The depth of the samples used in this paper is corrected for coring disturbance and gas expansion, based on layer-by-layer correlation and measurement on core photographs (Niitsuma, 


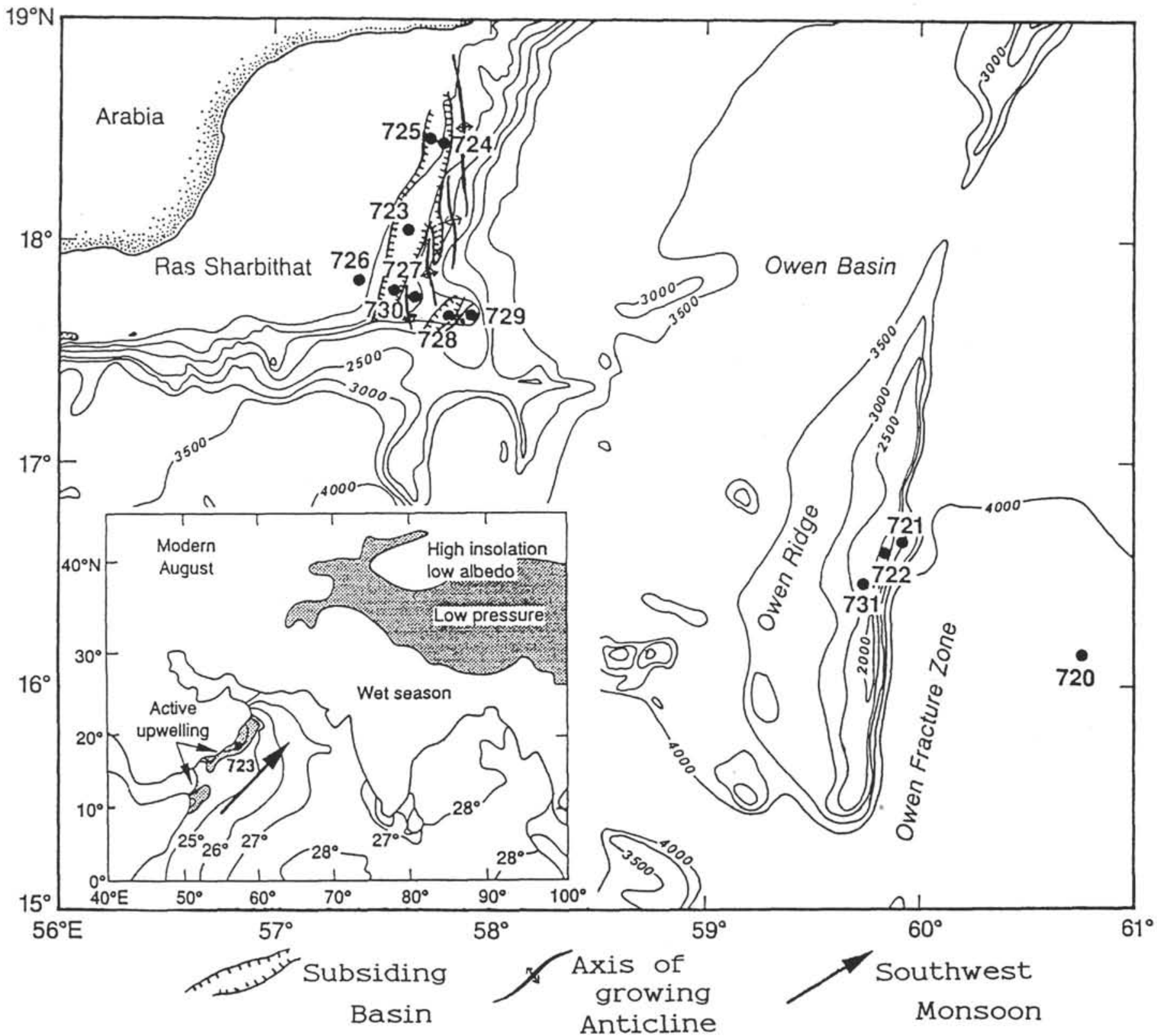

Figure 1. Location of Site 723 in the center of the subsiding basin in the Oman Margin and southwest monsoon (Prell, Niitsuma, et al., 1989).

this volume). Below $140 \mathrm{mbsf}$, ODP core log data were used for calculation of sample depth. In samples containing gas expansion voids, all the voids were closed and all separated sediment cores were pushed to the core top.

\section{ANALYTICAL METHOD}

The sediment sample was soaked in hot water for $12 \mathrm{hr}$ and wet-sieved through 200 mesh $(74 \mu \mathrm{m})$. The sieved sample was cleaned with ultrasonic wave and dried. Then the sample was dry-sieved through 64 mesh $(230 \mu \mathrm{m})$.

The tests of the following foraminiferal species were used for the isotopic analysis: planktonic, Pulleniatina obliquiloculata (Parker and Jones); benthic, Uvigerina excellens Todd.

Ten or more tests of the species, picked up under a binocular microscope, were put in a stainless-steel thimble. Methyl alcohol was dropped into the thimble, the tests were disaggregated with a thin needle and cleaned with ultrasonic wave, and the methyl alcohol was sucked out with a syringe and then evaporated.
The isotopic measurement followed the procedure by Wada et al. (1984). The carbonate tests were reacted in saturated pyrophosphoric acid at $60.00^{\circ} \mathrm{C}$, and the resulting $\mathrm{CO}_{2}$ gas was analyzed with MAT-250 and MAT-251 mass spectrometers modified for ultra-small sample analysis. The value thus obtained was converted into a value against a PDB standard by using NBS 20 . The converted values are $-4.18 \%$ for $\delta^{18} \mathrm{O}$ and $-1.07 \%$ for $\delta^{13} \mathrm{C}$ (Craig, 1957).

The precision for isotopic analyses is $0.02 \%$ for carbon and $0.05 \%$ for oxygen. Minimum volume for the isotopic analyses is $1 \mu \mathrm{L}$ for MAT-250 of Shizuoka and $2 \mu \mathrm{L}$ for MAT-251 of Kanazawa. The standard deviations of eight duplicated measurements on the isotopes for each individual test of foraminifer have ranges of $0.1 \%_{0}-0.27 \%_{0}$ for planktonic carbon, $0.18 \%_{0}-0.36 \%_{0}$ for planktonic oxygen, $0.09 \% 0-0.19 \%$ for benthic carbon, and $0.07 \%-0.28 \%$ for benthic oxygen.

The foraminiferal specimens for isotopic analysis can be picked up easily in the upper part of the sedimentary sequence, how- 


\section{Water Temperature $\left({ }^{\circ} \mathrm{C}\right)$ Oxgen $(\mathrm{ml} / \mathrm{I})$}

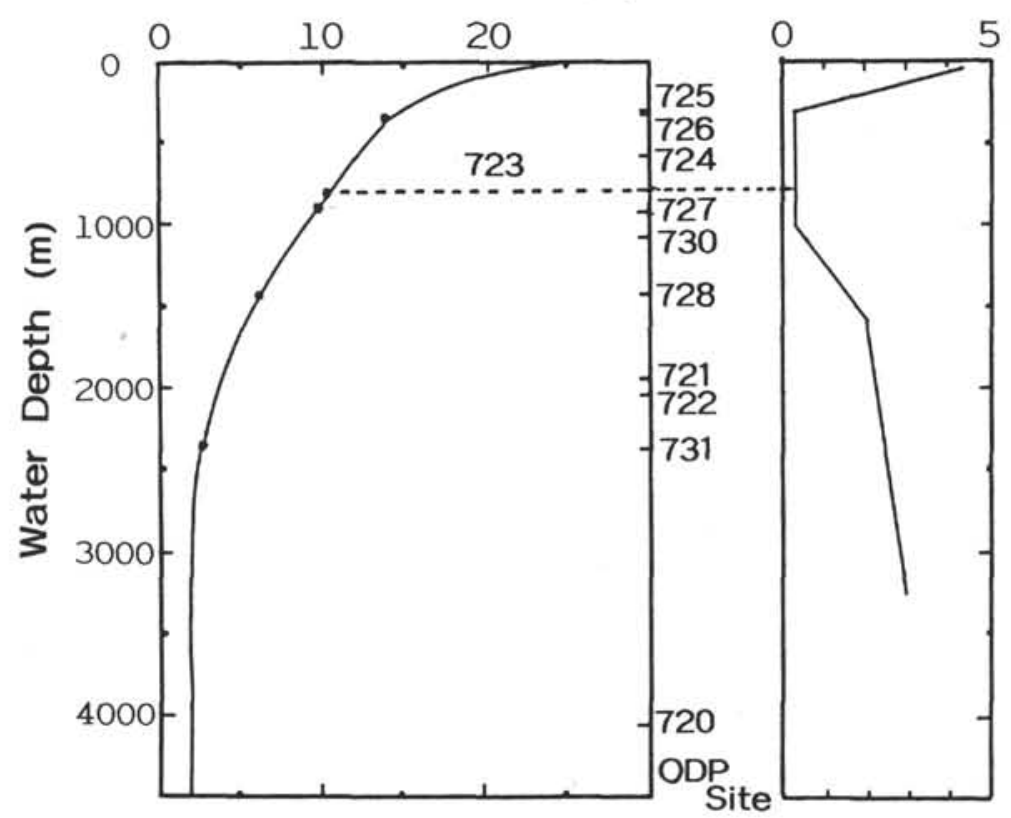

Figure 2. Depth profile of the measured temperature and dissolved oxygen (Prell, Niitsuma, et al., 1989) in the northwestern Indian Ocean, and ODP sites.

ever, specimens are less common in the lower part. Generally benthic foraminifers dominate in the dark colored sediment samples, and planktonic foraminifers in the white colored samples. Black samples in the lower part do not contain foraminiferal tests, but do contain fish teeth. The sample intervals in the lower part of the sequence are not close, compared with the upper part, because of the poor core recovery, gas expansion, and the nonpreservation of the foraminifers.

\section{OXYGEN ISOTOPE STRATIGRAPHY}

Oxygen isotope curve of planktonic $\delta^{18} \mathrm{O}_{\mathrm{P}}$ and benthic $\delta^{18} \mathrm{O}_{\mathrm{B}}$ from Site 723 (Figs. 3 and 4 and Table 1) can be correlated well with standard curves, for example, with Graphic Correlation Composite Records (Prell et al., 1986), SPECMAP (Imbrie et al., 1984), and Williams et al. (1988). Description and numerical coding of the oxygen isotope stratigraphic events in this paper follow Prell et al. (1986). Even numbered stages and substages represent glacial and/or colder intervals with heavier oxygen isotopic values, and odd numbered stages represent interglacial and/ or warmer intervals.

Oxygen isotope curve of planktonic $\delta^{18} \mathrm{O}_{\mathrm{P}}$ can be traced back continuously to Stage 23 with high resolution measurements. A significant difference appeared in Stage 15.4 with comparably heavy values, and a peak of Stage 15.5 is separated from the main Stage 15 peak. The oxygen isotope stratigraphic correlation can not be defined clearly in the time interval older than Stage 23. A tentative correlation is made to Stage 53 and shown in Figure 3.

The benthic foraminiferal curve $\delta^{18} \mathrm{O}_{\mathrm{B}}$ can also be correlated with the standard oxygen isotope stratigraphic curve, however, the amplitude of the fluctuations of the curve is significantly small, especially older than Stage 16. The maximum differences of benthic oxygen isotope values relative to planktonic differences between glacial and interglacial stages are $92.5 \%$ for Stages 1-2, $76.1 \%-89.0 \%$ for Stages $5-16,9.3 \%$ for Stages 17-18, and $38.4 \%$ for Stages 21-22.

Nannofossil biostratigraphic datums (Spaulding, this volume) can be located within the oxygen isotope stratigraphy (Figs. 3 and 4 and Table 1), last appearance of Helicosphaera inversa at
Stage 6.2, first appearance of Emiliania huxleyi at Stages 9.08.5, last appearance of Pseudoemiliania lacunosa at Stage 12.3, top acme of Reticulofenestra sp. A at Stages 23-25, first appearance of Gephyrocapsa parallela at Stages 27-29, last appearance of Gephyrocapsa (large) at Stages 34-35, and last appearance of Helicosphaera sellii at Stages 42-44.

\section{FLUCTUATION OF OXYGEN ISOTOPE RATIO}

The oxygen isotope values are controlled by oxygen isotopic composition and temperature of seawater. The contributions of the two factors can be quantified by comparing the oxygen isotope values of benthic and planktonic foraminifers. The difference of oxygen isotope $\delta^{18} \mathrm{O}_{\mathrm{B}-\mathrm{P}}$ is $2.3 \%_{0}$ in the Holocene sample of Site 723, corresponding to a temperature difference of about $10^{\circ} \mathrm{C}$. This is the same as the present temperature difference in the water column (Fig. 2), because the planktonic species Pulleniatina lives in the water depths from 25 to $74 \mathrm{~m}$ and precipitates its test under isotopic equilibrium (Kahn, 1979). In the case of Site 720 on the Indus Fan with water depth of $4045 \mathrm{~m}$, the isotope differences $\delta^{18} \mathrm{O}_{\mathrm{B}-\mathrm{P}}$ are $4 \%$ of which the corresponding temperature differences are $17^{\circ} \mathrm{C}$, and consistent also with present water column (Yasuda et al., this volume). The consistencies suggest that the isotope differences $\delta^{18} \mathrm{O}_{\mathrm{B}-\mathrm{P}}$ represent mainly temperature differences between the surface and bottom of the seawater in this area.

The oxygen isotope differences $\delta^{18} \mathrm{O}_{\mathrm{B}-\mathrm{P}}$ of Site 723 have a wide range from $1.5 \%$ to $3.5 \%$. Because the amplitude of the fluctuations of the benthic curve $\delta^{18} \mathrm{O}_{\mathrm{B}}$ is significantly less than that of the planktonic foraminifer $\delta^{18} \mathrm{O}_{\mathrm{P}}$ as mentioned above, the larger differences appear in the lighter oxygen isotope stages, i.e., in the interglacial stages. The amplitude of $\delta^{18} \mathrm{O}_{\mathrm{B}-\mathrm{P}}$ fluctuation for Stages 1-13 except the spikes is about $1 \%$, of which the equivalent temperature is $4^{\circ} \mathrm{C}$. The corresponding salinity difference is quite larger ( $4 \% 0)$, which is comparable to the influx of river water in the Bengal Bay (Duplessy, 1982).

The influence of the sea level change should count as a candidate for the explanation of the $\delta^{18} \mathrm{O}_{\mathrm{B}-\mathrm{P}}$ fluctuation; however, the measured temperature gradient is $0.8^{\circ} \mathrm{C} / 100 \mathrm{~m}$ for the depth 

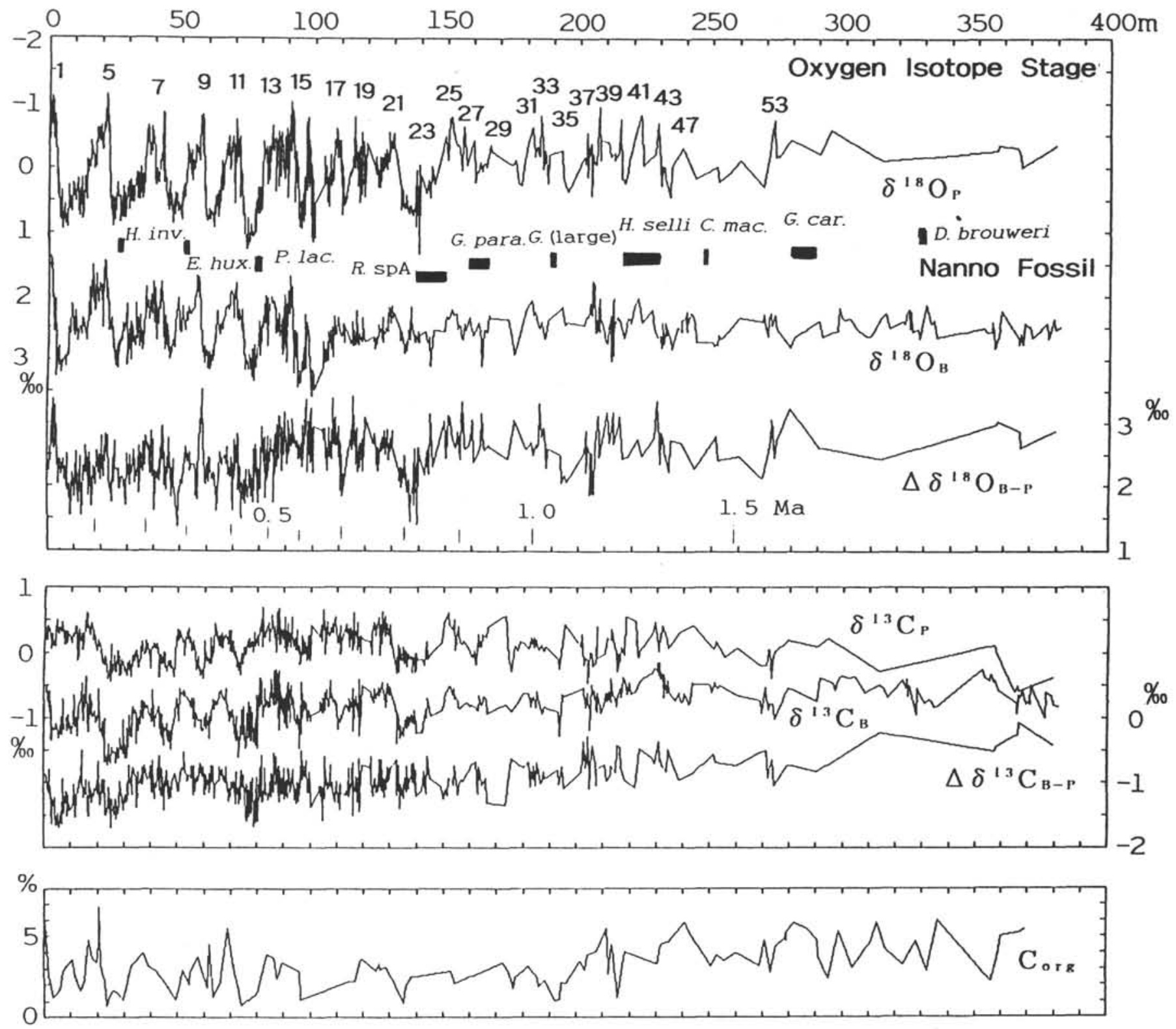

Figure 3. Isotopic results, organic carbon contents, and nannofossil biostratigraphic horizons of Site 723 in the Oman Margin. Number: oxygen isotope stratigraphic stages. $\delta^{18} \mathrm{O}_{\mathrm{P}}$ and $\delta^{13} \mathrm{C}_{\mathrm{P}}$ : oxygen and carbon isotope of planktonic foraminifer Pulleniatina obliquiloculata. $\delta^{18} \mathrm{O}_{\mathrm{B}}$ and $\delta^{13} \mathrm{C}_{\mathrm{B}}$ : oxygen and carbon isotope of benthic foraminifer Uvigerina excellens. $\delta^{18} \mathrm{O}_{\mathrm{B}-\mathrm{P}}$ and $\delta^{13} \mathrm{C}_{\mathrm{B}-\mathrm{P}}$ : difference of oxygen and carbon isotope between benthic $\delta^{18} \mathrm{O}_{\mathrm{B}}$ and $\delta^{13} \mathrm{C}_{\mathrm{B}}$, and planktonic $\delta^{18} \mathrm{O}_{\mathrm{P}}$ and $\delta^{13} \mathrm{C}_{\mathrm{p}}$. $\mathrm{C}_{\text {org }}$ : organic carbon content.

range from 330 to $800 \mathrm{~m}$ (Fig. 2) and the contribution of $100 \mathrm{~m}$ sea level change for the oxygen isotope is only $0.2 \%$.

Site 723 is located in the central part of the monsoonal upwelling area and the present SST (sea surface temperature) in summer is significantly cooled by the upwelling, $23^{\circ}-24^{\circ} \mathrm{C}$ which is almost same as the winter SST in this area (Prell and Hutson, 1979). The upwelling reduces the seasonal fluctuation of SST. SST for the Last Glacial Maximum, Stage 2, has been also estimated as almost the same as at present, because of cancelling the global cooling by the weakened monsoonal upwelling, based on isotopic and faunal data (Prell and Hutson, 1979). These data suggest that the oxygen isotope curve of planktonic $\delta^{18} \mathrm{O}_{\mathrm{P}}$ may represent rather the oxygen isotope fluctuation of seawater relating to the volume of global ice sheet, which is presented as the standard curves of oxygen isotope stratigraphy than the SST fluctuation, and the $\delta^{18} \mathrm{O}_{\mathrm{B}-\mathrm{P}}$ fluctuation should be mainly caused by the bottom water.

The $\delta^{18} \mathrm{O}_{\text {B-P }}$ fluctuation suggests that the bottom temperature was warmer and/or the isotopic composition of bottom water was affected by fresh water influx in the glacial stage.

Because the $800 \mathrm{~m}$ depth of Site 723 is in the center of the thermocline, the bottom temperature is sensitive to the position of the thermocline. During the strong upwelling stage, the thermocline would be pushed up and the bottom temperature should be cool, which is consistent with the cooler temperature in the interglacial stages with stronger southwest monsoon than in glacial stages.

Significant delays of benthic oxygen isotopes $\delta^{18} \mathrm{O}_{\mathrm{B}}$ from the planktonic $\delta^{18} \mathrm{O}_{\mathrm{P}}$ appear in the transgressive stages, Stages $10-9$, Stages 6-5, and Stages 2-1. The occurrence of the delay in the 

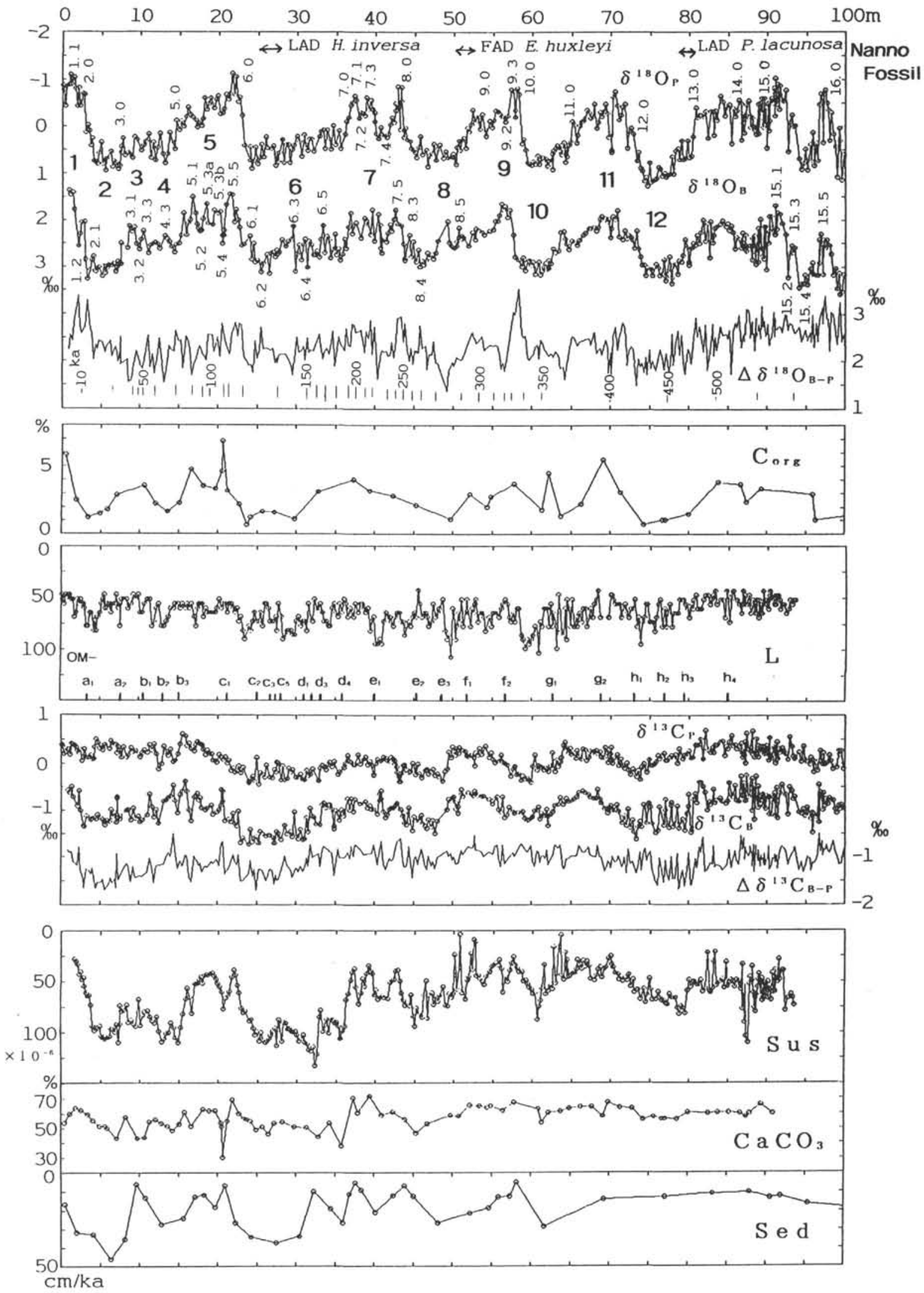

Figure 4. Isotopic results, nannofossil biostratigraphic horizons, and available data sets. Number: oxygen isotope stratigraphic stages. $\delta^{18} \mathrm{O}_{\mathrm{p}}$ and $\delta^{13} \mathrm{C}_{\mathrm{p}}$ : oxygen and carbon isotope of planktonic foraminifer Pulleniatina obliquiloculata. $\delta^{18} \mathrm{O}_{\mathrm{B}}$ and $\delta^{13} \mathrm{C}_{\mathrm{B}}$ : oxygen and carbon isotope of benthic foraminifer Uvigerina excellens. $\delta^{18} \mathrm{O}_{\mathrm{B}-\mathrm{P}}$ and $\delta^{13} \mathrm{C}_{\mathrm{B}-\mathrm{P}}$ : difference of oxygen and carbon isotope between benthic $\delta^{18} \mathrm{O}_{\mathrm{B}}$ and $\delta^{13} \mathrm{C}_{\mathrm{B}}$, and planktonic $\delta^{18} \mathrm{O}_{\mathrm{P}}$ and $\delta^{13} \mathrm{C}_{\mathrm{P}} . \mathrm{C}_{\mathrm{org}}$ : organic carbon content. L: lightness of sediment color (upside $=$ dark). Sus: magnetic susceptibility. $\mathrm{CaCO}_{3}$ : calcium carbonate content. Sed: rate of sedimentation. 
Table 1. Isotopic results of planktonic, Pulleniatina obliquiloculata $\left(\delta^{13} \mathrm{C}_{\mathrm{B}}, \delta^{18} \mathrm{O}_{\mathrm{P}}\right)$ and benthic foraminifers, Uvigerina excellens $\left(\delta^{13} C_{B}, \delta^{18} O_{P}\right)$ with remarks on the oxygen isotope stratigraphic horizon and nannofossil biostratigraphic horizon (Spaulding, this volume) at Site 723.

\begin{tabular}{|c|c|c|c|c|c|c|c|}
\hline \multirow{2}{*}{$\begin{array}{l}\text { Hole, core, section, } \\
\text { interval }(\mathrm{cm})\end{array}$} & \multirow{2}{*}{$\begin{array}{l}\text { Depth } \\
\text { (mbsf) }\end{array}$} & $\delta^{13} C_{\mathrm{P}}$ & $\delta^{18} \mathrm{O}_{\mathrm{P}}$ & $\delta^{13} \mathrm{C}_{\mathrm{B}}$ & $\delta^{13} \mathrm{O}_{\mathrm{B}}$ & \multirow{2}{*}{$\begin{array}{l}\delta^{18} \mathrm{O} \\
\text { Stage }\end{array}$} & \multirow{2}{*}{$\begin{array}{l}\text { Nannofossil } \\
\text { datum }\end{array}$} \\
\hline & & \multicolumn{4}{|c|}{$\%_{0}$ PDB } & & \\
\hline A-1H-1, 7 & 0.07 & 0.389 & -0.877 & & & 1.0 & \\
\hline A-1H-1, 21 & 0.21 & 0.357 & -0.777 & & & & \\
\hline A-1H-1, 41 & 0.41 & 0.209 & -0.451 & & & & \\
\hline A-1H-1, 61 & 0.61 & 0.231 & -0.858 & & & & \\
\hline A-1H-1, 81 & 0.81 & 0.300 & -0.892 & -0.563 & 1.364 & & \\
\hline $\mathrm{A}-1 \mathrm{H}-1,101$ & 1.01 & 0.295 & -1.101 & -0.618 & 1.455 & 1.1 & \\
\hline A-1H-1, 116 & 1.16 & 0.187 & -0.902 & & & & \\
\hline $\mathrm{A}-1 \mathrm{H}-1,131$ & 1.31 & 0.399 & -1.011 & -0.493 & 1.420 & & \\
\hline A-1H-1, 148 & 1.48 & 0.384 & -1.035 & -0.708 & 1.780 & & \\
\hline A-1H-2, 21 & 1.71 & 0.374 & -0.814 & & & & \\
\hline A-1H-2, 41 & 1.91 & 0.342 & -0.448 & & & & \\
\hline A-1H-2, 61 & 2.11 & 0.270 & -0.825 & -0.910 & 2.553 & 1.2 & \\
\hline A- $1 \mathrm{H}-2,81$ & 2.31 & 0.216 & -0.447 & -0.588 & 2.292 & & \\
\hline A-1H-2, 101 & 2.51 & 0.289 & -0.527 & -0.994 & 2.052 & & \\
\hline $\mathrm{A}-1 \mathrm{H}-2,116$ & 2.66 & 0.059 & -0.700 & & & 1.3 & \\
\hline $\mathrm{A}-1 \mathrm{H}-2,131$ & 2.81 & 0.130 & -0.687 & -1.330 & 2.041 & 2.0 & \\
\hline A-1H-2, 146 & 2.96 & 0.297 & 0.118 & -1.340 & 2.840 & 2.0 & \\
\hline A-1H-3, 21 & 3.21 & 0.147 & -0.029 & -1.118 & 3.256 & & \\
\hline A-1H-3, 41 & 3.41 & 0.113 & 0.086 & & & & \\
\hline A-1H-3, 61 & 3.61 & 0.134 & 0.414 & -1.164 & 3.070 & & \\
\hline A-1H-3, 78 & 3.78 & 0.177 & 0.252 & -1.193 & 2.857 & & \\
\hline $\mathrm{A}-1 \mathrm{H}-3,101$ & 4.01 & 0.087 & 0.746 & -1.159 & 2.779 & 2.1 & \\
\hline A-1H-3, 116 & 4.16 & 0.108 & 0.773 & & & & \\
\hline A-1H-3, 129 & 4.29 & 0.349 & 0.736 & -1.152 & 3.098 & & \\
\hline $\mathrm{A}-1 \mathrm{H}-3,148$ & 4.48 & 0.504 & 0.802 & -1.175 & 3.046 & & \\
\hline A-1H-4, 21 & 4.71 & 0.434 & 0.569 & -1.094 & 3.009 & & \\
\hline $\mathrm{A}-1 \mathrm{H}-4,41$ & 4.91 & 0.311 & 0.344 & & & & \\
\hline A-1H-4, 61 & 5.11 & 0.381 & 0.807 & -1.161 & 3.202 & & \\
\hline A- $1 \mathrm{H}-4,81$ & 5.31 & 0.288 & 0.720 & -1.189 & 3.002 & 2.2 & \\
\hline $\mathrm{A}-1 \mathrm{H}-4,101$ & 5.51 & 0.375 & 0.944 & -1.240 & 3.137 & & \\
\hline $\mathrm{A}-1 \mathrm{H}-4,116$ & 5.66 & 0.383 & 0.697 & -1.323 & 2.994 & & \\
\hline $\mathrm{A}-1 \mathrm{H}-4,131$ & 5.81 & & & -1.202 & 3.029 & & \\
\hline A-1H-4, 145 & 5.95 & & & -1.211 & 2.968 & & \\
\hline A-1H-5, 21 & 6.21 & 0.320 & 0.541 & -1.234 & 2.972 & & \\
\hline A- $1 \mathrm{H}-5,41$ & 6.41 & 0.461 & 0.859 & -0.976 & 2.960 & & \\
\hline A-1H-5, 61 & 6.61 & 0.414 & 0.741 & -1.137 & 2.942 & & \\
\hline A-1H-5, 81 & 6.81 & 0.408 & 0.792 & -1.047 & 3.104 & & \\
\hline A-1H-5, 101 & 7.01 & 0.397 & 0.849 & -1.250 & 3.018 & & \\
\hline A-1H-5, 116 & 7.16 & 0.227 & 0.904 & -0.712 & 2.893 & & \\
\hline A-1H-5, 116 & 7.16 & & & -0.737 & 2.958 & & \\
\hline A-1H-5, 131 & 7.31 & 0.341 & 0.853 & -1.145 & 2.917 & & \\
\hline A-1H-5, 148 & 7.48 & 0.275 & 0.434 & -1.184 & 2.940 & 3.0 & \\
\hline $\mathrm{A}-2 \mathrm{H}-1,31$ & 7.51 & 0.301 & 0.565 & -1.116 & 2.496 & 3.0 & \\
\hline $\mathrm{A}-2 \mathrm{H}-1,51$ & 7.71 & 0.136 & 0.253 & & & & \\
\hline A-2H-1, 81 & 8.01 & 0.339 & 0.624 & & & & \\
\hline $\mathrm{A}-2 \mathrm{H}-1,116$ & 8.36 & 0.143 & 0.560 & -0.994 & 2.599 & & \\
\hline $\mathrm{A}-2 \mathrm{H}-1,141$ & 8.61 & 0.191 & 0.583 & -0.974 & 2.133 & & \\
\hline A- $2 \mathrm{H}-2,21$ & 8.91 & 0.329 & 0.669 & -1.196 & 2.256 & 3.1 & \\
\hline A- $2 \mathrm{H}-2,51$ & 9.21 & 0.260 & 0.224 & -0.952 & 2.154 & & \\
\hline A- $2 \mathrm{H}-2,81$ & 9.51 & 0.276 & 0.281 & -1.167 & 2.506 & 3.2 & \\
\hline $\mathrm{A}-2 \mathrm{H}-2,116$ & 9.86 & & & -1.124 & 2.626 & & \\
\hline $\mathrm{A}-2 \mathrm{H}-2,141$ & 10.11 & 0.154 & 0.514 & -1.245 & 2.415 & & \\
\hline A- $2 \mathrm{H}-3,21$ & 10.41 & 0.217 & 0.400 & -1.196 & 2.238 & 3.3 & \\
\hline A- $2 \mathrm{H}-3,51$ & 10.71 & 0.288 & 0.333 & & & & \\
\hline A-2H-3, 81 & 11.01 & 0.236 & 0.171 & -1.082 & 2.712 & 4.0 & \\
\hline A-2H-3, 116 & 11.36 & 0.389 & 0.673 & -0.639 & 2.534 & 4.0 & \\
\hline $\mathrm{A}-2 \mathrm{H}-3,141$ & 11.61 & 0.277 & 0.353 & -0.921 & 2.512 & & \\
\hline A-2H-4, 21 & 11.91 & 0.404 & 0.729 & -1.127 & 2.482 & & \\
\hline $\mathrm{A}-2 \mathrm{H}-4,53$ & 12.23 & 0.203 & 0.452 & -0.982 & 2.508 & & \\
\hline A-2H-4, 81 & 12.51 & -0.105 & 0.143 & -0.269 & 2.617 & & \\
\hline $\mathrm{A}-2 \mathrm{H}-4,116$ & 12.86 & -0.012 & 0.594 & & & & \\
\hline A-2H- $-4,141$ & 13.11 & 0.358 & 0.794 & -0.798 & 2.346 & 4.3 & \\
\hline A- $2 \mathrm{H}-5,21$ & 13.41 & 0.253 & 0.588 & -0.765 & 2.400 & & \\
\hline A-2H-5, 51 & 13.71 & 0.182 & 0.136 & -0.725 & 2.458 & & \\
\hline A-2H-5, 81 & 14.01 & 0.302 & 0.323 & -0.695 & 2.573 & & \\
\hline A-2H-5, 116 & 14.36 & 0.033 & 0.485 & -0.469 & 2.694 & 4.4 & \\
\hline A- $2 \mathrm{H}-5,141$ & 14.61 & 0.078 & -0.127 & -0.785 & 2.519 & 5.0 & \\
\hline A-2H-6, 21 & 14.91 & 0.215 & 0.072 & -1.035 & 2.489 & & \\
\hline A- $2 \mathrm{H}-6,53$ & 15.23 & 0.409 & -0.116 & -0.665 & 2.326 & & \\
\hline A-2H-6, 81 & 15.51 & 0.622 & 0.003 & -0.584 & 1.862 & & \\
\hline A-2H-6, 116 & 15.86 & 0.580 & -0.210 & -0.376 & 2.315 & & \\
\hline A-2H-6, 141 & 16.11 & 0.374 & -0.411 & -0.590 & 2.066 & & \\
\hline A- $2 \mathrm{H}-7,18$ & 16.38 & 0.427 & -0.270 & -0.981 & 1.989 & & \\
\hline A- $2 \mathrm{H}-7,51$ & 16.71 & 0.314 & -0.198 & -1.210 & 1.503 & 5.1 & \\
\hline
\end{tabular}


Table 1 (continued).

\begin{tabular}{|c|c|c|c|c|c|c|c|}
\hline \multirow{2}{*}{$\begin{array}{l}\text { Hole, core, section, } \\
\text { interval }(\mathrm{cm})\end{array}$} & \multirow{2}{*}{$\begin{array}{l}\text { Depth } \\
\text { (mbsf) }\end{array}$} & $\delta^{13} \mathrm{C}_{\mathrm{P}}$ & $\delta^{18} \mathrm{O}_{\mathrm{P}}$ & $\delta^{13} \mathrm{C}_{\mathrm{B}}$ & $\delta^{13} \mathrm{O}_{\mathrm{B}}$ & \multirow{2}{*}{$\begin{array}{l}\delta^{18} \mathrm{O} \\
\text { Stage }\end{array}$} & \multirow{2}{*}{$\begin{array}{c}\text { Nannofossil } \\
\text { datum }\end{array}$} \\
\hline & & \multicolumn{4}{|c|}{$\%_{0}$ PDB } & & \\
\hline B-3H-3, 21 & 17.01 & 0.274 & -0.137 & -0.712 & 1.862 & & \\
\hline B-3H-3, 41 & 17.21 & 0.397 & 0.019 & -0.669 & 2.086 & & \\
\hline B-3H-3, 63 & 17.43 & 0.452 & -0.062 & -0.633 & 2.168 & & \\
\hline B- $3 \mathrm{H}-3,81$ & 17.61 & 0.425 & -0.083 & -0.765 & 2.243 & 5.2 & \\
\hline B-3H-3, 101 & 17.81 & 0.401 & -0.020 & -0.725 & 2.226 & & \\
\hline A- $3 \mathrm{H}-1,16$ & 17.96 & 0.389 & -0.167 & -0.940 & 1.983 & & \\
\hline A- $3 \mathrm{H}-1,41$ & 18.21 & 0.300 & -0.612 & & & & \\
\hline A- $3 \mathrm{H}-1,66$ & 18.46 & 0.190 & -0.367 & -0.988 & 1.651 & $5.3 \mathrm{a}$ & \\
\hline A-3H-1, 91 & 18.71 & 0.281 & -0.488 & -0.951 & 2.070 & & \\
\hline A-3H-1, 116 & 18.96 & 0.211 & -0.611 & & & & \\
\hline A-3H-1, 141 & 19.21 & 0.215 & -0.462 & -0.884 & 2.096 & & \\
\hline A-3H- 2,16 & 19.46 & 0.067 & -0.407 & -1.073 & 1.800 & & \\
\hline A-3H-2, 41 & 19.71 & 0.075 & -0.584 & & & & \\
\hline A-3H-2, 64 & 19.94 & 0.030 & -0.655 & -1.025 & 1.853 & $5.3 \mathrm{~b}$ & \\
\hline A-3H-2, 91 & 20.21 & 0.191 & -0.272 & -0.987 & 1.819 & & \\
\hline A- $3 \mathrm{H}-2,116$ & 20.46 & & & -0.890 & 2.321 & & \\
\hline A- $3 \mathrm{H}-2,116$ & 20.46 & 0.153 & -0.296 & -0.550 & 2.496 & 5.4 & \\
\hline A- $3 \mathrm{H}-2,141$ & 20.71 & 0.086 & -0.378 & -0.586 & 2.220 & & \\
\hline A-3H-2, 141 & 20.71 & & & -0.792 & 2.331 & & \\
\hline A- $3 \mathrm{H}-3,16$ & 20.96 & 0.140 & -0.696 & -1.206 & 1.670 & & \\
\hline A- $3 \mathrm{H}-3,41$ & 21.21 & 0.016 & -0.585 & & & & \\
\hline A- $3 \mathrm{H}-3,66$ & 21.46 & 0.066 & -0.704 & -1.181 & 1.438 & 5.5 & \\
\hline A-3H-3, 91 & 21.71 & -0.145 & -1.139 & -1.243 & 1.456 & & \\
\hline A- $3 \mathrm{H}-3,116$ & 21.96 & -0.093 & -0.689 & -1.050 & 2.038 & & \\
\hline A- $3 \mathrm{H}-3,141$ & 22.21 & -0.169 & -1.056 & -1.267 & 1.779 & & \\
\hline A- $3 \mathrm{H}-4,16$ & 22.46 & -0.161 & -0.534 & -1.338 & 1.990 & & \\
\hline A- $3 \mathrm{H}-4,41$ & 22.71 & -0.059 & -0.636 & -1.017 & 2.161 & & . \\
\hline A-3H-4, 64 & 22.94 & -0.181 & -0.249 & -1.685 & 2.535 & 60 & $\theta$ \\
\hline A-3H-4, 91 & 23.21 & -0.082 & 0.408 & -1.588 & 2.510 & 0.0 & \\
\hline A- $3 \mathrm{H}-4,116$ & 23.46 & & & & & & \\
\hline A-3H-5, 16 & 23.96 & -0.407 & 0.729 & & & & \\
\hline A-3H-5, 16 & 23.96 & -0.376 & 0.443 & -1.722 & 2.349 & 6.1 & \\
\hline A- 3 H- 5,41 & 24.21 & -0.328 & 0.900 & -1.412 & 2.823 & & \\
\hline A- $3 \mathrm{H}-5,65$ & 24.45 & -0.262 & 0.735 & -1.671 & 2.499 & & 1 \\
\hline A-3H-5, 91 & 24.71 & -0.352 & 0.458 & -1.609 & 2.890 & & \\
\hline A-3H-5, 116 & 24.96 & 0.137 & 0.806 & -1.567 & 2.859 & & \\
\hline A-3H-5, 141 & 25.21 & -0.436 & 0.670 & -1.696 & 2.912 & & \\
\hline A-3H- 6,16 & 25.46 & -0.183 & 0.393 & -1.447 & 3.110 & 6.2 & \\
\hline A- 3 H- $-6,41$ & 25.71 & -0.212 & 0.655 & -1.499 & 2.926 & & LAD of \\
\hline A-3H- 6,66 & 25.96 & -0.281 & 0.221 & & & & H. inversa \\
\hline A-3H-6, 91 & 26.21 & -0.039 & 0.455 & -1.541 & 2.740 & & \\
\hline A- 3 H- 6,116 & 26.46 & & & -1.522 & 3.132 & & \\
\hline A-3H-6-141 & 26.71 & -0.280 & 0.470 & -1.537 & 2.705 & & \\
\hline A-3H- 7,14 & 26.94 & -0.299 & 0.419 & -1.582 & 2.695 & & 1 \\
\hline A- $3 \mathrm{H}-7,41$ & 27.21 & -0.131 & 0.831 & -1.702 & 2.790 & & \\
\hline A-3H-7, 61 & 27.41 & -0.177 & 0.582 & -1.559 & 2.645 & & \\
\hline B- $4 \mathrm{H}-3,43$ & 27.53 & 0.023 & 0.764 & -1.459 & 2.718 & & \\
\hline B- $4 \mathrm{H}-3,65$ & 27.75 & 0.003 & 0.564 & -1.491 & 2.694 & & \\
\hline A- $4 \mathrm{H}-1,16$ & 28.06 & -0.334 & 0.287 & -1.627 & 2.426 & & \\
\hline A- $4 \mathrm{H}-1,41$ & 28.31 & -0.059 & 0.778 & & & & \\
\hline A- $4 \mathrm{H}-1,66$ & 28.56 & -0.334 & 0.399 & -1.317 & 2.551 & & \\
\hline A- 4 H- 1,91 & 28.81 & -0.312 & 0.389 & -1.493 & 2.448 & & \\
\hline A-4H-1, 116 & 29.06 & -0.095 & 0.770 & & & & \\
\hline A- $4 \mathrm{H}-1,141$ & 29.31 & -0.178 & 0.494 & -1.457 & 2.403 & & \\
\hline A- $4 \mathrm{H}-2,16$ & 29.56 & -0.128 & 0.410 & -1.556 & 2.131 & 6.3 & \\
\hline A- $4 \mathrm{H}-2,41$ & 29.81 & & & -1.027 & 3.085 & & \\
\hline A- 4 H- 2,62 & 30.02 & -0.273 & 0.215 & -1.496 & 2.729 & & \\
\hline A- $4 \mathrm{H}-2,91$ & 30.31 & -0.287 & 0.329 & -1.603 & 2.520 & & \\
\hline A- $-4 \mathrm{H}-2,116$ & 30.56 & -0.225 & 0.630 & -1.412 & 2.842 & & \\
\hline A-4H-2, 141 & 30.81 & -0.322 & 0.185 & -1.625 & 2.509 & & \\
\hline A- $4 \mathrm{H}-3,16$ & 31.06 & -0.346 & 0.391 & -1.610 & 2.407 & & \\
\hline A- $-4 \mathrm{H}-3,41$ & 31.31 & -0.139 & 0.509 & -1.156 & 3.004 & 6.4 & \\
\hline A- $-4 \mathrm{H}-3,66$ & 31.56 & -0.257 & 0.264 & -1.457 & 2.448 & & \\
\hline A- $4 \mathrm{H}-3,91$ & 31.81 & -0.226 & 0.269 & -0.956 & 2.523 & & \\
\hline A- $-4 \mathrm{H}-3,116$ & 32.06 & -0.114 & 0.531 & -1.124 & 2.746 & & \\
\hline A- $4 \mathrm{H}-3,141$ & 32.31 & & & -1.132 & 2.736 & & \\
\hline A. $-4 \mathrm{H}-4,16$ & 32.56 & & & -1.290 & 2.663 & & \\
\hline A- $4 \mathrm{H}-4,41$ & 32.81 & -0.350 & 0.214 & -1.274 & 2.781 & & \\
\hline A- $4 \mathrm{H}-4,62$ & 33.02 & -0.102 & 0.098 & -1.302 & 2.401 & & \\
\hline $\mathrm{A}-4 \mathrm{H}-4,91$ & 33.31 & -0.030 & 0.069 & -0.870 & 2.116 & 6.5 & \\
\hline $\mathrm{A}-4 \mathrm{H}-4,116$ & 33.56 & -0.068 & 0.470 & -0.835 & 2.686 & & \\
\hline $\mathrm{A}-4 \mathrm{H}-4,141$ & 33.81 & -0.121 & 0.066 & -0.937 & 2.349 & & \\
\hline A-4H-5, 16 & 34.06 & -0.025 & 0.267 & -0.881 & 2.419 & & \\
\hline A- 4 H- 5,41 & 34.31 & 0.021 & 0.481 & -0.867 & 2.794 & & \\
\hline A- 4 H-5, 66 & 34.56 & -0.062 & -0.013 & -0.965 & 2.635 & & \\
\hline A- 4 H-5, 91 & 34.81 & 0.040 & 0.399 & -1.337 & 2.299 & & \\
\hline A- 4 H- 5,91 & 34.81 & & & -1.385 & 2.583 & & \\
\hline
\end{tabular}


Table 1 (continued).

\begin{tabular}{|c|c|c|c|c|c|c|c|}
\hline \multirow{2}{*}{$\begin{array}{l}\text { Hole, core, section, } \\
\text { interval }(\mathrm{cm})\end{array}$} & \multirow{2}{*}{$\begin{array}{l}\text { Depth } \\
\text { (mbsf) }\end{array}$} & $\delta^{13} C_{P}$ & $\delta^{18} \mathrm{O}_{\mathrm{P}}$ & $\delta^{13} \mathrm{C}_{\mathrm{B}}$ & $\delta^{13} \mathrm{O}_{\mathrm{B}}$ & \multirow{2}{*}{$\begin{array}{l}\delta^{18} \mathrm{O} \\
\text { Stage }\end{array}$} & \multirow{2}{*}{$\begin{array}{l}\text { Nannofossil } \\
\text { datum }\end{array}$} \\
\hline & & \multicolumn{4}{|c|}{$\%_{00}$ PDB } & & \\
\hline A- $4 \mathrm{H}-5,116$ & 35.06 & & & -1.003 & 2.733 & & \\
\hline A-4H-5, 141 & 35.31 & -0.224 & 0.095 & -1.051 & 2.615 & & \\
\hline $\mathrm{A}-4 \mathrm{H}-6,16$ & 35.56 & -0.176 & 0.461 & -1.200 & 2.846 & 6.6 & \\
\hline A-4H-6, 41 & 35.81 & & & -0.981 & 2.729 & & \\
\hline A-4H-6, 66 & 36.06 & -0.086 & 0.163 & -1.106 & 2.399 & & \\
\hline A-4H-6, 91 & 36.31 & 0.006 & -0.108 & -0.993 & 2.595 & & \\
\hline A-4H-6, 116 & 36.56 & 0.170 & -0.201 & -0.732 & 2.141 & 7.0 & \\
\hline A- $4 \mathrm{H}-6,141$ & 36.81 & 0.062 & -0.463 & -0.974 & 1.858 & & \\
\hline $\mathrm{A}-4 \mathrm{H}-7,16$ & 37.06 & 0.032 & -0.526 & -0.743 & 2.275 & & \\
\hline A- $4 \mathrm{H}-7,41$ & 37.31 & -0.019 & -0.654 & -1.013 & 2.108 & 7.1 & \\
\hline A- $4 \mathrm{H}-7,64$ & 37.54 & -0.022 & -0.563 & -0.750 & 2.047 & & \\
\hline A-5H-1, 31 & 37.91 & 0.199 & -0.190 & & & 7.2 & \\
\hline A-5H-1, 65 & 38.15 & -0.009 & -0.306 & -0.847 & 2.368 & & \\
\hline A-5H-1, 91 & 38.39 & 0.065 & -0.288 & & & & \\
\hline A-5H-1, 121 & 38.69 & 0.102 & -0.241 & -0.849 & 1.962 & & \\
\hline A-5H-1, 141 & 38.89 & 0.145 & -0.636 & -0.800 & 2.008 & 7.3 & \\
\hline A-5H-2, 16 & 39.14 & -0.015 & -0.387 & -0.942 & 2.094 & & \\
\hline A-5H-2, 41 & 39.39 & 0.048 & -0.567 & -0.885 & 2.302 & & \\
\hline A-5H-2, 63 & 39.61 & -0.038 & -0.385 & -0.964 & 1.777 & & \\
\hline A-5H-2, 91 & 39.89 & -0.233 & -0.314 & -0.969 & 2.428 & & \\
\hline A-5H-2, 91 & 39.89 & -0.212 & -0.106 & & & & \\
\hline A-5H-2, 116 & 40.14 & -0.004 & 0.147 & & & & \\
\hline A-5H-2, 141 & 40.39 & 0.104 & 0.250 & -1.056 & 1.877 & & \\
\hline A- $5 \mathrm{H}-3,16$ & 40.64 & 0.053 & 0.004 & -0.774 & 2.099 & & \\
\hline A-5H-3, 16 & 40.64 & & & -0.638 & 2.594 & & \\
\hline A-5H-3, 41 & 40.89 & 0.070 & 0.163 & -0.574 & 2.413 & & \\
\hline A-5H-3, 41 & 40.89 & & & -0.738 & 2.694 & & \\
\hline A-5H-3, 66 & 41.14 & 0.142 & 0.188 & -1.004 & 2.387 & & \\
\hline A- $5 \mathrm{H}-3,91$ & 41.39 & 0.125 & 0.221 & -1.134 & 2.451 & 7.4 & \\
\hline A-5H-3, 116 & 41.64 & 0.067 & 0.146 & -1.075 & 2.265 & & \\
\hline A-5H-3, 141 & 41.89 & 0.048 & -0.046 & -0.963 & 2.087 & & \\
\hline A- $5 \mathrm{H}-4,16$ & 42.14 & 0.145 & -0.200 & -0.932 & 2.223 & & \\
\hline A- $5 \mathrm{H}-4,41$ & 42.39 & 0.110 & -0.097 & -0.941 & 1.980 & & \\
\hline A- $5 \mathrm{H}-4,63$ & 42.61 & 0.073 & -0.347 & & & & \\
\hline A- $5 \mathrm{H}-4,63$ & 42.61 & -0.011 & -0.509 & -0.910 & 1.781 & 7.5 & \\
\hline A- $5 \mathrm{H}-4,91$ & 42.89 & -0.174 & -0.853 & -0.835 & 2.070 & & \\
\hline A-5H-4, 91 & 42.89 & -0.070 & -0.351 & & & & \\
\hline A-5H-4, 116 & 43.12 & -0.370 & 0.052 & & & & \\
\hline A-5H-4, 116 & 43.12 & -0.270 & -0.468 & & & & \\
\hline A-5H-4, 139 & 43.33 & -0.197 & -0.546 & & & & \\
\hline A-5H-4, 139 & 43.33 & -0.371 & -0.835 & -0.968 & 2.121 & 8.0 & \\
\hline A-5H-5, 16 & 43.60 & -0.174 & 0.231 & & & 0.0 & \\
\hline A-5H-5, 16 & 43.60 & -0.029 & 0.065 & -1.099 & 2.488 & & \\
\hline A-5H-5, 41 & 43.85 & & & -1.042 & 2.797 & & \\
\hline A-5H-5, 41 & 43.85 & -0.028 & 0.029 & -1.248 & 2.841 & & \\
\hline A-5H-5, 66 & 44.09 & -0.107 & 0.288 & -1.308 & 2.535 & & \\
\hline A- $5 \mathrm{H}-5,91$ & 44.34 & -0.166 & 0.312 & -0.939 & 2.316 & 8.3 & \\
\hline A- $5 \mathrm{H}-5,116$ & 44.59 & -0.159 & 0.469 & -1.224 & 2.770 & & \\
\hline A- $5 \mathrm{H}-5,141$ & 44.82 & 0.012 & 0.503 & -1.417 & 2.445 & & \\
\hline A-5H-6, 19 & 45.08 & -0.051 & 0.546 & -1.116 & 2.791 & & \\
\hline A-5H- 6,41 & 45.28 & -0.218 & 0.649 & -1.191 & 2.877 & & \\
\hline A-5H-6, 66 & 45.51 & 0 & 0.441 & -1.161 & 2.636 & & \\
\hline A-5H-6, 92 & 45.75 & -0.199 & 0.212 & -1.188 & 2.986 & 8.4 & \\
\hline A-5H-6, 116 & 45.98 & -0.274 & 0.586 & -1.089 & 2.864 & & \\
\hline A-5H-6, 141 & 46.21 & & & -1.265 & 2.944 & & \\
\hline A- $5 \mathrm{H}-7,16$ & 46.46 & -0.131 & 0.506 & -1.337 & 2.840 & & \\
\hline A- $5 \mathrm{H}-7,41$ & 46.71 & -0.110 & 0.852 & -1.373 & 2.688 & & \\
\hline A- $5 \mathrm{H}-7,59$ & 46.89 & -0.199 & 0.621 & -1.173 & 2.785 & & \\
\hline B-6H-2, 141 & 47.21 & -0.173 & 0.561 & -1.352 & 2.723 & & \\
\hline B-6H-3, 16 & 47.46 & -0.115 & 0.380 & -1.194 & 2.809 & & \\
\hline B- $6 \mathrm{H}-3,41$ & 47.71 & -0.238 & 0.717 & -1.482 & 2.696 & & \\
\hline B- $6 \mathrm{H}-3,68$ & 47.98 & -0.207 & 0.468 & -1.208 & 2.436 & & \\
\hline B- $6 \mathrm{H}-3,91$ & 48.21 & -0.270 & 0.390 & & & & \\
\hline B-6H-3, 141 & 48.71 & -0.363 & 0.668 & & & & \\
\hline B- $6 \mathrm{H}-4,16$ & 48.96 & -0.161 & 0.531 & & & & \\
\hline B- $6 \mathrm{H}-4,41$ & 49.21 & -0.093 & 0.644 & -0.960 & 2.026 & & \\
\hline A- $6 \mathrm{H}-1,12$ & 49.42 & 0.253 & 0.629 & -0.732 & 2.458 & & \\
\hline A-6H-1, 41 & 49.69 & 0.127 & 0.649 & -0.778 & 2.541 & & \\
\hline A-6H-1, 68 & 49.86 & 0.354 & 0.577 & -0.710 & 2.573 & & \\
\hline A-6H-1, 91 & 50.08 & 0.282 & 0.677 & -0.686 & 2.581 & & 1 \\
\hline A- $6 \mathrm{H}-1,116$ & 50.26 & 0.194 & 0.803 & & & & \\
\hline A- $6 \mathrm{H}-1,141$ & 50.41 & & & -0.954 & 2.447 & & \\
\hline A- $6 \mathrm{H}-1,141$ & 50.41 & 0.330 & 0.435 & -0.806 & 2.534 & & \\
\hline A- $6 \mathrm{H}-2,16$ & 50.64 & 0.223 & 0.294 & -0.898 & 2.347 & & \\
\hline A- $6 \mathrm{H}-2,16$ & 50.64 & & & -1.043 & 2.165 & 8.5 & \\
\hline A- $6 \mathrm{H}-2,29$ & 50.77 & 0.342 & 0.494 & & & & \\
\hline
\end{tabular}


Table 1 (continued).

\begin{tabular}{|c|c|c|c|c|c|c|c|}
\hline \multirow{2}{*}{$\begin{array}{l}\text { Hole, core, section, } \\
\text { interval }(\mathrm{cm})\end{array}$} & \multirow{2}{*}{$\begin{array}{l}\text { Depth } \\
\text { (mbsf) }\end{array}$} & $\delta^{13} \mathrm{C}_{\mathrm{P}}$ & $\delta^{18} \mathrm{O}_{\mathrm{P}}$ & $\delta^{13} \mathrm{C}_{\mathrm{B}}$ & $\delta^{13} \mathrm{O}_{\mathrm{B}}$ & \multirow{2}{*}{$\begin{array}{l}\delta^{18} \mathrm{O} \\
\text { Stage }\end{array}$} & \multirow{2}{*}{$\begin{array}{l}\text { Nannofossil } \\
\text { datum }\end{array}$} \\
\hline & & \multicolumn{4}{|c|}{$\% 0$ PDB } & & \\
\hline A- $6 \mathrm{H}-2,69$ & 51.02 & 0.306 & 0.285 & -0.537 & 2.328 & & FAD \\
\hline A- $6 \mathrm{H}-2,69$ & 51.02 & & & -0.624 & 2.386 & & E. huxleyi \\
\hline A- $6 \mathrm{H}-2,91$ & 51.23 & 0.278 & 0.238 & -0.670 & 2.355 & & \\
\hline A- $6 \mathrm{H}-2,116$ & 51.48 & 0.265 & 0.449 & & & & \\
\hline A- $6 \mathrm{H}-2,142$ & 51.71 & 0.172 & 0.026 & & & & \\
\hline A- $6 \mathrm{H}-3,16$ & 51.93 & 0.159 & 0.106 & -0.607 & 2.542 & & \\
\hline A- $6 \mathrm{H}-3,41$ & 52.15 & 0.191 & -0.050 & & & & \\
\hline A- $6 \mathrm{H}-3,66$ & 52.38 & 0.180 & -0.374 & -0.627 & 2.257 & & \\
\hline A- $6 \mathrm{H}-3,91$ & 52.56 & 0.135 & -0.192 & -0.662 & 2.308 & & \\
\hline A- $6 \mathrm{H}-3,121$ & 52.76 & -0.038 & -0.095 & -0.574 & 2.399 & & \\
\hline A- $6 \mathrm{H}-3,141$ & 52.89 & 0.231 & -0.218 & -0.613 & 2.176 & & \\
\hline A- $6 \mathrm{H}-4,16$ & 53.13 & 0.189 & -0.149 & & & & \\
\hline A- $6 \mathrm{H}-4,16$ & 53.13 & 0.190 & -0.213 & -0.656 & 2.213 & & \\
\hline A- $6 \mathrm{H}-4,41$ & 53.38 & 0.325 & 0.047 & & & & \\
\hline A- $6 \mathrm{H}-4,63$ & 53.58 & 0.201 & -0.278 & -0.678 & 2.258 & & \\
\hline A- $6 \mathrm{H}-4,91$ & 53.86 & 0.307 & 0.044 & -0.682 & 2.292 & 9.0 & \\
\hline A- $6 \mathrm{H}-4,116$ & 54.11 & 0.368 & 0.200 & & & 9.0 & \\
\hline A- $6 \mathrm{H}-5,16$ & 54.61 & 0.150 & -0.126 & -0.735 & 2.226 & & \\
\hline A- $6 \mathrm{H}-5,41$ & 54.84 & 0.239 & 0.005 & & & & \\
\hline A- 6 H- 5,66 & 55.08 & 0.104 & -0.141 & -0.837 & 2.197 & & \\
\hline A- $6 \mathrm{H}-5,91$ & 55.33 & 0.020 & -0.355 & -0.737 & 2.006 & 9.1 & \\
\hline A- $6 \mathrm{H}-5,118$ & 55.60 & 0.052 & -0.336 & -0.697 & 2.004 & & \\
\hline A- $6 \mathrm{H}-5,141$ & 55.83 & 0.072 & -0.298 & -0.959 & 1.885 & & \\
\hline A- $6 \mathrm{H}-6,16$ & 56.08 & 0.192 & -0.225 & -1.006 & 1.635 & & \\
\hline A- $6 \mathrm{H}-6,41$ & 56.33 & 0.045 & -0.220 & -0.694 & 1.768 & & \\
\hline A- $6 \mathrm{H}-6,66$ & 56.58 & 0.008 & -0.109 & -1.138 & 1.697 & 9.2 & \\
\hline A-6H-6, 91 & 56.83 & -0.016 & -0.253 & -1.073 & 1.931 & & \\
\hline A- $6 \mathrm{H}-6,118$ & 57.10 & & & -0.922 & 1.781 & & \\
\hline A- $6 \mathrm{H}-6,141$ & 57.33 & -0.251 & -0.790 & & & & \\
\hline A- $6 \mathrm{H}-7,16$ & 57.58 & -0.111 & -0.760 & -1.006 & 2.291 & & \\
\hline A- $6 \mathrm{H}-7,41$ & 57.82 & -0.067 & -0.362 & & & & \\
\hline A- $6 \mathrm{H}-7,41$ & 57.82 & -0.059 & -0.214 & -1.048 & 2.773 & & \\
\hline A- $6 \mathrm{H}-7,66$ & 58.06 & -0.217 & -0.820 & & & 9.3 & \\
\hline A- $6 \mathrm{H}-7,87$ & 58.25 & -0.199 & -0.706 & -1.024 & 2.832 & & \\
\hline B-7H-3, 66 & 58.64 & -0.345 & 0.367 & -1.041 & 2.960 & 10.0 & \\
\hline B-7H-3, 91 & 58.89 & -0.252 & 0.268 & -1.005 & 2.783 & & \\
\hline B-7H-3, 116 & 59.14 & -0.310 & 0.500 & -1.147 & 3.054 & & \\
\hline B-7H-3, 144 & 59.42 & -0.298 & 0.773 & -1.191 & 2.859 & & \\
\hline B- $7 \mathrm{H}-4,16$ & 59.64 & -0.337 & 0.806 & -1.172 & 2.847 & & \\
\hline B-7H-4, 41 & 59.89 & -0.384 & 0.813 & -1.141 & 2.934 & & \\
\hline B-7H-4, 61 & 60.09 & -0.046 & 0.744 & -1.123 & 2.869 & & \\
\hline B-7H-4, 89 & 60.37 & 0.191 & 0.804 & -0.995 & 3.128 & & \\
\hline A-7H-1, 16 & 60.66 & -0.120 & 0.618 & -0.985 & 2.982 & & \\
\hline A-7H-1, 35 & 60.85 & -0.037 & 0.807 & -0.924 & 2.875 & & \\
\hline A-7H-2, 16 & 61.06 & -0.051 & 0.687 & -1.160 & 3.143 & & \\
\hline A-7H-2, 38 & 61.28 & -0.068 & 0.645 & -1.045 & 3.071 & & \\
\hline A-7H-2, 66 & 61.48 & -0.122 & 0.707 & -1.083 & 2.983 & & \\
\hline A-7H-2, 91 & 61.70 & -0.076 & 0.681 & -1.105 & 2.908 & & \\
\hline A-7H-2, 121 & 61.92 & -0.115 & 0.840 & -0.964 & 3.032 & & \\
\hline A-7H-2, 141 & 62.12 & -0.160 & 0.723 & -1.320 & 2.843 & & \\
\hline A-7H-2, 141 & 62.12 & & & -1.110 & 3.016 & & \\
\hline A-7H-3, 16 & 62.35 & -0.030 & 0.715 & -1.079 & 2.905 & & \\
\hline A- $7 \mathrm{H}-3,47$ & 62.53 & -0.078 & 0.581 & & & & \\
\hline A- $7 \mathrm{H}-3,47$ & 62.53 & 0.231 & 0.905 & & & & \\
\hline A-7H-3, 66 & 62.71 & -0.043 & 0.507 & -1.008 & 2.606 & & \\
\hline A-7H-3, 91 & 62.94 & -0.164 & 0.446 & -0.890 & 2.678 & & \\
\hline A-7H-3, 121 & 63.19 & 0.083 & 0.411 & & & & \\
\hline A-7H-3, 142 & 63.37 & -0.046 & 0.502 & -0.862 & 2.645 & & \\
\hline A- $7 \mathrm{H}-4,16$ & 63.58 & 0.037 & 0.405 & -0.702 & 2.258 & & \\
\hline A- $7 \mathrm{H}-4,41$ & 63.73 & 0.146 & 0.584 & & & & \\
\hline A- $7 \mathrm{H}-4,66$ & 63.97 & 0.385 & 0.349 & -0.857 & 2.243 & & \\
\hline A-7H-4, 91 & 64.15 & 0.454 & 0.751 & -0.799 & 2.382 & & \\
\hline A-7H-4, 127 & 64.39 & 0.350 & 0.415 & & & & \\
\hline A-7H-4, 145 & 64.42 & 0.299 & 0.641 & -0.727 & 2.489 & & \\
\hline A-7H-5, 16 & 64.63 & 0.234 & 0.389 & -0.802 & 2.621 & & \\
\hline A-7H-5, 41 & 64.88 & 0.336 & 0.324 & & & 110 & \\
\hline A-7H-5, 68 & 65.09 & 0.209 & -0.115 & -0.760 & 2.429 & 11.0 & \\
\hline A-7H-5, 91 & 65.29 & 0.230 & -0.039 & -0.735 & 2.469 & & \\
\hline A-7H-5, 126 & 65.59 & 0.110 & 0.343 & & & & \\
\hline A-7H- 5,141 & 65.74 & 0.287 & 0.177 & -0.686 & 2.522 & & \\
\hline A-7H- 6,16 & 65.99 & 0.162 & -0.013 & -0.599 & 2.422 & & \\
\hline A-7H- 6,41 & 66.24 & 0.103 & -0.118 & & & & \\
\hline A-7H- 6,66 & 66.49 & 0.320 & -0.130 & -0.571 & 2.312 & & \\
\hline A-7H- 6,91 & 66.74 & 0.301 & -0.206 & -0.596 & 2.274 & & \\
\hline A-7H-6, 116 & 66.97 & 0.072 & -0.365 & & & & \\
\hline A-7H-6, 141 & 67.22 & 0.146 & -0.312 & -0.642 & 2.180 & & \\
\hline
\end{tabular}


Table 1 (continued).

\begin{tabular}{|c|c|c|c|c|c|c|c|}
\hline \multirow{2}{*}{$\begin{array}{l}\text { Hole, core, section, } \\
\text { interval }(\mathrm{cm})\end{array}$} & \multirow{2}{*}{$\begin{array}{l}\text { Depth } \\
\text { (mbsf) }\end{array}$} & $\delta^{13} \mathrm{C}_{\mathrm{P}}$ & $\delta^{18} \mathrm{O}_{\mathrm{P}}$ & $\delta^{13} \mathrm{C}_{\mathrm{B}}$ & $\delta^{13} \mathrm{O}_{\mathrm{B}}$ & \multirow{2}{*}{$\begin{array}{l}\delta^{18} \mathrm{O} \\
\text { Stage }\end{array}$} & \multirow{2}{*}{$\begin{array}{l}\text { Nannofossil } \\
\text { datum }\end{array}$} \\
\hline & & \multicolumn{4}{|c|}{$\%_{0}$ PDB } & & \\
\hline A-7H-7, 16 & 67.47 & 0.272 & -0.181 & -0.690 & 2.176 & & \\
\hline A-7H-7, 41 & 67.70 & 0.240 & -0.210 & & & & \\
\hline A-7H-7, 66 & 67.95 & 0.269 & -0.484 & -0.721 & 2.141 & & \\
\hline A-7H-7, 91 & 68.17 & 0.285 & 0.027 & -0.717 & 2.214 & & \\
\hline A-7H-7, 116 & 68.39 & 0.217 & -0.095 & -0.544 & 2.151 & & \\
\hline A-7H-7, 141 & 68.62 & 0.259 & -0.321 & -0.736 & 1.971 & & \\
\hline A-7H-8, 16 & 68.87 & 0.150 & -0.229 & -0.832 & 1.919 & & \\
\hline A-7H- 8,41 & 69.12 & 0.133 & -0.332 & & & & \\
\hline A-7H-8, 66 & 69.37 & 0.341 & -0.498 & -0.958 & 1.958 & & \\
\hline A- 7 H- 8,89 & 69.60 & 0.234 & -0.300 & -0.944 & 2.017 & & \\
\hline B-8H-3, 31 & 69.75 & 0.031 & 0.219 & -1.049 & 1.979 & & \\
\hline B-8H-3, 66 & 69.96 & 0.208 & 0.156 & -0.928 & 2.355 & & \\
\hline B- $8 \mathrm{H}-3,83$ & 70.13 & 0.050 & 0.498 & & & & \\
\hline B- $8 \mathrm{H}-3,83$ & 70.13 & 0.339 & 0.540 & -0.885 & 2.170 & & \\
\hline B- $8 \mathrm{H}-3,111$ & 70.31 & 0.010 & -0.637 & -0.976 & 1.921 & & \\
\hline В-8H-3, 139 & 70.59 & 0.123 & -0.754 & -0.981 & 1.983 & & \\
\hline B- $8 \mathrm{H}-4,11$ & 70.81 & 0.197 & -0.464 & -0.876 & 1.791 & & \\
\hline A- $8 \mathrm{H}-1,16$ & 71.06 & -0.037 & -0.150 & -1.004 & 2.347 & & \\
\hline A- $8 \mathrm{H}-1,41$ & 71.31 & 0.108 & -0.266 & & & & \\
\hline A- $8 \mathrm{H}-1,66$ & 71.56 & -0.033 & -0.433 & -1.248 & 2.397 & & \\
\hline A- $8 \mathrm{H}-1,91$ & 71.81 & -0.096 & -0.495 & -1.102 & 2.216 & & \\
\hline A- $8 \mathrm{H}-1,116$ & 72.06 & 0.013 & 0.447 & -1.322 & 2.382 & & \\
\hline A- $8 \mathrm{H}-1,141$ & 72.31 & -0.132 & 0.088 & -0.973 & 2.319 & & \\
\hline A- $8 \mathrm{H}-2,16$ & 72.56 & -0.225 & 0.020 & -1.361 & 2.346 & & \\
\hline A- $8 \mathrm{H}-2,41$ & 72.81 & -0.113 & 0.149 & -1.189 & 2.421 & & \\
\hline A- 8 H- $-2,63$ & 73.03 & & & -1.451 & 2.580 & & \\
\hline A- 8 H- 2,91 & 73.30 & -0.269 & 0.726 & -1.609 & 2.225 & & \\
\hline A-8H-2, 116 & 73.54 & -0.137 & 0.538 & -1.347 & 2.603 & 12.0 & \\
\hline A- $8 \mathrm{H}-2,141$ & 73.77 & -0.331 & 0.874 & -1.224 & 2.765 & 12.0 & \\
\hline A- $8 \mathrm{H}-3,15$ & 74.01 & -0.066 & 0.939 & -1.340 & 2.930 & & \\
\hline A- $8 \mathrm{H}-3,41$ & 74.24 & -0.007 & 1.157 & -0.874 & 2.933 & & \\
\hline A-8H-3, 66 & 74.47 & -0.098 & 1.076 & -0.916 & 3.171 & & \\
\hline A- $8 \mathrm{H}-3,91$ & 74.71 & -0.172 & 1.265 & -1.252 & 3.004 & & \\
\hline A- $8 \mathrm{H}-3,116$ & 74.94 & 0.081 & 0.780 & -0.836 & 3.042 & & \\
\hline A- $8 \mathrm{H}-3,141$ & 75.14 & -0.040 & 1.187 & -0.923 & 3.033 & & \\
\hline A- $8 \mathrm{H}-4,16$ & 75.38 & -0.036 & 1.146 & -1.276 & 3.169 & & \\
\hline A- $8 \mathrm{H}-4,41$ & 75.63 & -0.001 & 1.122 & & & & \\
\hline A- $8 \mathrm{H}-4,62$ & 75.84 & 0.047 & 1.091 & -1.466 & 2.920 & & \\
\hline A- $8 \mathrm{H}-4,91$ & 76.13 & 0.179 & 0.859 & -1.420 & 2.991 & & \\
\hline A- $8 \mathrm{H}-4,113$ & 76.33 & 0.104 & 0.902 & -0.920 & 3.164 & & \\
\hline A-8H-4, 141 & 76.56 & 0.181 & 1.055 & -1.075 & 3.139 & & \\
\hline A-8H-5, 16 & 76.81 & 0.220 & 0.987 & -1.320 & 2.831 & & \\
\hline A- $8 \mathrm{H}-5,41$ & 77.06 & & & -0.757 & 3.174 & & \\
\hline A- $8 \mathrm{H}-5,41$ & 77.06 & 0.075 & 1.070 & -0.986 & 3.266 & & \\
\hline A- 8 H- 5,65 & 77.27 & 0.076 & 1.037 & -1.255 & 3.054 & & \\
\hline A-8H-5, 91 & 77.53 & 0.135 & 1.004 & -1.360 & 2.764 & & \\
\hline A- $8 \mathrm{H}-5,116$ & 77.78 & 0.175 & 0.632 & -0.817 & 3.215 & & \\
\hline A- $8 \mathrm{H}-5,116$ & 77.78 & & & -0.884 & 3.328 & & \\
\hline A- $8 \mathrm{H}-5,141$ & 78.03 & 0.125 & 1,006 & -1.340 & 2.932 & & \\
\hline A- $8 \mathrm{H}-6,16$ & 78.28 & 0.194 & 0.509 & -1.271 & 2.926 & & \\
\hline A- 8 H- 6,41 & 78.53 & 0.013 & 0.476 & -0.909 & 3.142 & & \\
\hline A- 8 H- 6,66 & 78.78 & 0.310 & 0.700 & -1.378 & 2.849 & & \\
\hline A-8H-6, 91 & 79.03 & 0.192 & 0.304 & -1.227 & 2.873 & & LAD \\
\hline A-8H-6, 116 & 79.28 & 0.094 & 0.703 & -1.182 & 2.737 & & P. lacunosa \\
\hline A- $8 \mathrm{H}-6,141$ & 79.53 & 0.193 & 0.660 & -1.417 & 2.401 & & \\
\hline A- $8 \mathrm{H}-7,16$ & 79.78 & 0.205 & 0.297 & -1.059 & 2.574 & & \\
\hline A- $8 \mathrm{H}-7,41$ & 80.03 & 0.266 & 0.658 & -0.646 & 2.875 & & \\
\hline A- $8 \mathrm{H}-7,41$ & 80.03 & & & -0.905 & 2.933 & & \\
\hline A- 8 H- 7,66 & 80.28 & 0.122 & 0.628 & -1.469 & 2.582 & & \\
\hline A- $8 \mathrm{H}-7,85$ & 80.47 & 0.138 & 0.216 & -1.393 & 2.566 & & \\
\hline B-9H-4, 92 & 80.57 & & & -0.745 & 2.487 & 13.0 & \\
\hline B-9H-4, 116 & 80.81 & 0.394 & -0.419 & -0.638 & 2.513 & 13.0 & \\
\hline B-9H-4, 141 & 81.06 & 0.393 & -0.209 & -0.836 & 2.319 & & \\
\hline B-9H-5, 16 & 81.31 & 0.403 & -0.266 & -0.392 & 2.487 & & \\
\hline B-9H-5, 41 & 81.56 & 0.514 & -0.268 & -0.394 & 2.259 & & \\
\hline B-9H-5, 66 & 81.81 & 0.106 & -0.224 & -0.476 & 1.992 & & \\
\hline B-9H-5, 91 & 82.06 & 0.701 & -0.339 & -0.603 & 2.439 & & \\
\hline A-9H-1, 16 & 82.36 & 0.294 & 0.243 & -0.977 & 2.064 & & \\
\hline A-9H-1, 54 & 82.64 & & & -0.794 & 2.778 & & \\
\hline A-9H-1, 66 & 82.76 & 0.232 & -0.333 & -0.876 & 2.020 & & \\
\hline A-9H-1, 91 & 83.01 & 0.346 & -0.332 & -0.805 & 2.470 & & \\
\hline A-9H-1, 106 & 83.16 & 0.237 & 0.186 & -0.539 & 2.315 & & \\
\hline A-9H-1, 141 & 83.38 & 0.395 & -0.359 & -0.823 & 2.165 & & \\
\hline A-9H-2, 16 & 83.57 & 0.397 & -0.134 & -0.760 & 2.123 & & \\
\hline A-9H-2, 63 & 84.04 & 0.457 & -0.662 & -0.726 & 2.089 & & \\
\hline A-9H-2, 91 & 84.30 & 0.470 & -0.539 & -0.800 & 2.011 & & \\
\hline A-9H-2, 116 & 84.55 & 0.180 & -0.244 & & & & \\
\hline
\end{tabular}


Table 1 (continued).

\begin{tabular}{|c|c|c|c|c|c|c|c|}
\hline \multirow{2}{*}{$\begin{array}{l}\text { Hole, core, section, } \\
\text { interval }(\mathrm{cm})\end{array}$} & \multirow{2}{*}{$\begin{array}{l}\text { Depth } \\
\text { (mbsf) }\end{array}$} & $\delta^{13} \mathrm{C}_{\mathrm{P}}$ & $\delta^{18} \mathrm{O}_{\mathrm{P}}$ & $\delta^{13} \mathrm{C}_{\mathrm{B}}$ & $\delta^{13} \mathrm{O}_{\mathrm{B}}$ & \multirow{2}{*}{$\begin{array}{l}\delta^{18} \mathrm{O} \\
\text { Stage }\end{array}$} & \multirow{2}{*}{$\begin{array}{l}\text { Nannofossil } \\
\text { datum }\end{array}$} \\
\hline & & \multicolumn{4}{|c|}{$\%_{0}$ PDB } & & \\
\hline A-9H-2, 141 & 84.75 & 0.356 & -0.450 & -0.846 & 2.131 & & \\
\hline A-9H-3, 16 & 84.93 & 0.314 & -0.412 & -0.784 & 2.072 & & \\
\hline A- 9 H- 3,40 & 85.17 & 0.447 & -0.477 & -0.404 & 2.199 & & \\
\hline A-9H-3, 67 & 85.44 & 0.583 & 0.366 & -0.652 & 2.118 & & \\
\hline A-9H-3, 91 & 85.65 & 0.371 & -0.397 & -0.689 & 2.251 & 14.0 & \\
\hline A-9H-3, 116 & 85.89 & 0.396 & -0.233 & -0.470 & 2.599 & 14.0 & \\
\hline A-9H-3, 141 & 86.14 & 0.318 & -0.361 & -0.764 & 2.365 & & \\
\hline A-9H-4, 16 & 86.38 & 0.360 & -0.567 & -0.795 & 2.410 & & \\
\hline A-9H $-4,40$ & 86.62 & 0.427 & -0.487 & & & & \\
\hline B-10X-1, 16 & 86.66 & 0.279 & 0.280 & -0.262 & 2.583 & & \\
\hline A-9H-4, 67 & 86.82 & 0.128 & -0.283 & -0.680 & 2.265 & & \\
\hline B-10X-1, 41 & 86.86 & 0.203 & -0.087 & -0.650 & 2.460 & & \\
\hline A- $9 \mathrm{H}-4,95$ & 87.08 & 0.172 & 0.137 & -0.413 & 2.567 & & \\
\hline B- $10 X-1,66$ & 87.11 & 0.456 & -0.178 & -0.585 & 2.617 & & \\
\hline A-9H-4, 116 & 87.27 & 0.003 & -0.266 & -0.786 & 2.308 & & \\
\hline B-10X-1, 91 & 87.34 & 0.639 & -0.476 & -0.253 & 2.646 & & \\
\hline B- $10 X-1,116$ & 87.59 & 0.299 & -0.555 & -0.685 & 2.545 & & \\
\hline A- $9 \mathrm{H}-5,33$ & 87.83 & 0.264 & -0.233 & -0.597 & 2.538 & & \\
\hline B-10X-1, 141 & 87.84 & 0.347 & 0.012 & -0.430 & 2.519 & & \\
\hline B- $10 X-2,16$ & 88.09 & 0.675 & -0.023 & -0.307 & 2.836 & & \\
\hline A- $9 \mathrm{H}-5,66$ & 88.10 & 0.214 & -0.201 & -0.990 & 2.373 & & \\
\hline B-10X-2, 41 & 88.31 & 0.277 & -0.006 & -0.468 & 2.603 & & \\
\hline A-9H-5, 91 & 88.35 & 0.128 & 0.068 & -1.174 & 2.322 & & \\
\hline B- $10 X-2,64$ & 88.54 & 0.505 & -0.016 & -0.260 & 2.919 & & \\
\hline A-9H-5, 116 & 88.60 & 0.198 & 0.157 & -0.662 & 2.687 & & \\
\hline A-9H-5, 141 & 88.85 & 0.153 & -0.484 & -0.933 & 2.178 & & \\
\hline B-10X-2, 105 & 88.89 & 0.381 & 0.152 & -0.472 & 2.878 & & \\
\hline B- $10 X-2,116$ & 89.00 & 0.270 & -0.151 & -0.688 & 2.653 & & \\
\hline A-9H- 6,16 & 89.10 & 0.353 & -0.514 & -0.846 & 1.981 & & \\
\hline B- $10 X-3,41$ & 89.22 & 0.301 & -0.318 & -0.661 & 2.583 & & \\
\hline A- $9 \mathrm{H}-6,40$ & 89.34 & 0.306 & -0.609 & -0.754 & 2.573 & & \\
\hline B- $10 X-3,92$ & 89.41 & 0.357 & 0.013 & -0.653 & 2.751 & & \\
\hline A-9H- 6,66 & 89.60 & 0.271 & -0.620 & -0.898 & 2.134 & & \\
\hline B- $10 X-3,116$ & 89.65 & 0.206 & -0.059 & -0.726 & 2.820 & & \\
\hline A-9H- 6,91 & 89.83 & 0.217 & -0.412 & -0.952 & 2.126 & & \\
\hline B- $10 \times-4,16$ & 89.95 & 0.373 & 0.451 & -0.678 & 3.040 & 150 & \\
\hline A-9H-6, 116 & 90.08 & 0.312 & -0.504 & -0.740 & 2.291 & 15.0 & \\
\hline B-10X-4, 41 & 90.20 & 0.067 & 0.091 & -0.708 & 2.474 & & \\
\hline B- $10 \times-4,62$ & 90.24 & 0.540 & -0.127 & -0.687 & 2.232 & & \\
\hline A-9H-6, 141 & 90.33 & 0.181 & -0.532 & -1.079 & 1.912 & & \\
\hline A-9H-7, 16 & 90.58 & 0.104 & -0.611 & -1.073 & 1.915 & & \\
\hline B-10X-4, 116 & 90.66 & 0.197 & -0.514 & -0.825 & 2.154 & & \\
\hline A-9H- 7,41 & 90.83 & 0.338 & -0.824 & -0.705 & 2.220 & & \\
\hline B- $10 X-5,16$ & 90.93 & 0.314 & -0.246 & -0.620 & 2.292 & & \\
\hline B- $10 X-5,41$ & 91.03 & 0.271 & -0.632 & -0.738 & 2.295 & & \\
\hline A-9H-7, 66 & 91.08 & 0.007 & -1.022 & -1.098 & 1.691 & 151 & \\
\hline B- $10 X-5,66$ & 91.28 & 0.447 & -0.487 & -0.510 & 2.185 & 15.1 & \\
\hline A-9H-7, 89 & 91.31 & 0.095 & -0.899 & -1.154 & 1.836 & & \\
\hline B-10X-5, 101 & 91.48 & 0.321 & -0.383 & & & & \\
\hline B-10X-5, 116 & 91.62 & 0.235 & -0.874 & -0.613 & 1.873 & & \\
\hline B-10X-5, 141 & 91.87 & 0.327 & -0.569 & -0.448 & 2.069 & & \\
\hline B- $10 X-6,16$ & 92.12 & 0.253 & -0.557 & & & & \\
\hline B- $10 X-6,41$ & 92.37 & 0.210 & -0.788 & -0.653 & 2.291 & & \\
\hline B- $10 X-6,66$ & 92.62 & 0.089 & 0.523 & -1.071 & 3.205 & 15.2 & \\
\hline B-10X-6, 91 & 92.87 & 0.561 & -0.095 & -0.488 & 2.716 & & \\
\hline B- $10 X-6,116$ & 93.12 & 0.358 & -0.260 & -0.571 & 2.538 & & \\
\hline B- $10 X-6,141$ & 93.37 & 0.161 & -0.002 & -0.739 & 2.692 & & \\
\hline B- $10 X-7,10$ & 93.56 & 0.099 & -0.016 & -0.695 & 2.590 & & \\
\hline B-11X-1, 16 & 94.06 & 0.245 & 0.689 & -1.030 & 3.423 & & \\
\hline B-11X-1, 70 & 94.37 & 0.044 & 0.918 & -1.054 & 3.345 & & \\
\hline B- $11 X-1,101$ & 94.68 & 0.353 & 0.794 & -0.962 & 3.351 & & \\
\hline B-11X-1, 116 & 94.83 & 0.087 & 0.483 & -1.107 & 3.161 & & \\
\hline B-11X-1, 141 & 95.05 & 0.046 & 0.734 & -1.047 & 3.270 & & \\
\hline B-11X-2, 8 & 95.19 & 0.042 & 0.920 & -0.942 & 3.367 & & \\
\hline B- $11 X-2,41$ & 95.49 & -0.024 & 0.389 & -1.060 & 3.026 & & \\
\hline B-11X-2, 96 & 95.61 & 0.133 & 0.725 & -1.159 & 3.148 & & \\
\hline B-11X-2, 118 & 95.81 & 0.069 & 0.139 & -1.037 & 3.122 & & \\
\hline $\mathrm{A}-11 \mathrm{X}-1,105$ & 95.85 & & & -1.465 & 2.908 & & \\
\hline A-11X-1, 116 & 95.96 & -0.176 & 0.460 & & & & \\
\hline B-11X-2, 141 & 96.04 & 0.054 & 0.831 & -1.068 & 3.146 & & \\
\hline B- $11 X-3,41$ & 96.27 & -0.070 & 0.355 & -1.130 & 3.168 & & \\
\hline B-11X-3, 66 & 96.50 & -0.143 & 0.724 & -1.086 & 3.164 & & \\
\hline B- $11 X-3,84$ & 96.67 & 0.056 & -0.472 & -0.449 & 2.425 & & \\
\hline A-11X-2, 40 & 96.70 & & & -1.244 & 2.914 & & \\
\hline B-11X-3, 122 & 96.83 & 0.276 & -0.704 & -0.598 & 2.298 & & \\
\hline B-11X-3, 141 & 97.02 & -0.003 & -0.539 & -0.651 & 2.591 & & \\
\hline A-11X-2, 89 & 97.19 & -0.023 & 0.354 & -0.980 & 3.152 & & \\
\hline
\end{tabular}


Table 1 (continued).

\begin{tabular}{|c|c|c|c|c|c|c|c|}
\hline \multirow{2}{*}{$\begin{array}{l}\text { Hole, core, section, } \\
\text { interval }(\mathrm{cm})\end{array}$} & \multirow{2}{*}{$\begin{array}{l}\text { Depth } \\
\text { (mbsf) }\end{array}$} & $\delta^{13} \mathrm{C}_{\mathrm{P}}$ & $\delta^{18} \mathrm{O}_{\mathrm{P}}$ & $\delta^{13} \mathrm{C}_{\mathrm{B}}$ & $\delta^{13} \mathrm{O}_{\mathrm{B}}$ & \multirow{2}{*}{$\begin{array}{l}\delta^{18} \mathrm{O} \\
\text { Stage }\end{array}$} & \multirow{2}{*}{$\begin{array}{l}\text { Nannofossil } \\
\text { datum }\end{array}$} \\
\hline & & \multicolumn{4}{|c|}{$\%_{0}$ PDB } & & \\
\hline B-11X-4, 19 & 97.25 & 0.228 & -0.704 & -0.608 & 2.417 & & \\
\hline B- $11 X-4,41$ & 97.44 & -0.026 & -0.782 & -0.889 & 2.597 & & \\
\hline B-11X-4, 64 & 97.66 & 0.014 & -0.502 & -0.733 & 2.419 & & \\
\hline B-11X-4, 116 & 97.78 & 0.020 & -0.376 & -0.854 & 2.532 & & \\
\hline B-11X-4, 139 & 98.01 & 0.184 & 0.308 & -0.724 & 2.536 & & \\
\hline B-11X-5, 39 & 98.21 & 0.057 & -0.308 & -0.761 & 2.730 & 16.0 & \\
\hline B-11X-5, 90 & 98.52 & -0.119 & 0.170 & -0.772 & 3.171 & & \\
\hline B-11X-5, 116 & 98.78 & -0.050 & 0.441 & -0.960 & 3.031 & & \\
\hline B-11X-5, 141 & 98.96 & -0.078 & 1.081 & -1.037 & 3.451 & & \\
\hline B-11X-6, 16 & 99.21 & 0.264 & 0.035 & -0.885 & 3.138 & & \\
\hline B-11X-6, 54 & 99.29 & 0.193 & 0.323 & -0.960 & 3.570 & & \\
\hline B-11X-6, 81 & 99.56 & 0.273 & 1.137 & -0.872 & 3.550 & & \\
\hline B-11X-6, 116 & 99.81 & -0.106 & 0.603 & -0.935 & 3.166 & & \\
\hline B-11X- 6,141 & 100.02 & 0.007 & 1.078 & -1.035 & 3.292 & & \\
\hline B-11X-7, 16 & 100.20 & 0.168 & 0.967 & -0.970 & 3.481 & & \\
\hline B-11X-7, 34 & 100.38 & 0.414 & 0.540 & -0.965 & 3.486 & & \\
\hline B- $12 X-1,16$ & 103.56 & 0.234 & 0.254 & -0.892 & 3.134 & & \\
\hline B- $12 X-1,41$ & 103.73 & 0.312 & -0.188 & -0.737 & 2.726 & & \\
\hline B-12X-1, 66 & 103.93 & 0.442 & 0.155 & -0.771 & 2.911 & & \\
\hline B-12X-1, 91 & 104.17 & 0.413 & 0.151 & -0.806 & 3.060 & & \\
\hline B-12X-1, 116 & 104.34 & 0.175 & -0.032 & -0.876 & 2.885 & & \\
\hline B-12X-1, 141 & 104.59 & 0.660 & 0.535 & -0.925 & 3.043 & & \\
\hline B-12X-2, 16 & 104.81 & 0.646 & 0.505 & -0.671 & 2.952 & & \\
\hline B- $12 X-2,41$ & 105.01 & 0.340 & 0.130 & -0.737 & 2.723 & & \\
\hline B-12X-2, 63 & 105.23 & 0.421 & 0.434 & -0.837 & 2.788 & & \\
\hline B-12X-2, 89 & 105.49 & 0.350 & 0.596 & -0.897 & 3.007 & & \\
\hline B-12X-3, 16 & 105.70 & -0.020 & 0.335 & -0.604 & 2.578 & 17.0 & \\
\hline B- $12 X-3,41$ & 105.95 & 0.313 & -0.210 & -0.687 & 2.719 & 17.0 & \\
\hline B-12X-3, 66 & 106.20 & 0.487 & 0.276 & -0.554 & 2.763 & & \\
\hline B-12X-3, 91 & 106.45 & 0.333 & 0.078 & -0.599 & 2.545 & & \\
\hline B-12X-3, 116 & 106.70 & 0.289 & 0.216 & -0.827 & 2.840 & & \\
\hline B-12X-3, 141 & 106.95 & 0.256 & 0.178 & -0.799 & 2.821 & & \\
\hline B-12X-4, 16 & 107.18 & 0.255 & 0.036 & -0.761 & 2.473 & & \\
\hline B- $12 X-4,41$ & 107.43 & 0.385 & -0.426 & -0.584 & 2.959 & & \\
\hline B-12X-4, 64 & 107.66 & 0.292 & -0.333 & -0.418 & 2.323 & & \\
\hline B-12X-4, 91 & 107.93 & 0.346 & -0.315 & -0.693 & 2.361 & & \\
\hline B-12X-4, 116 & 108.18 & 0.455 & -0.611 & -0.617 & 2.431 & & \\
\hline B-12X-4, 141 & 108.43 & 0.305 & -0.484 & -0.688 & 2.617 & & \\
\hline B-12X-5, 16 & 108.68 & 0.410 & -0.150 & -0.758 & 2.494 & & \\
\hline B- $12 X-5,41$ & 108.93 & & & -0.716 & 2.470 & & \\
\hline B-12X-5, 66 & 109.18 & 0.553 & -0.170 & -0.759 & 2.486 & & \\
\hline B- $12 X-5,91$ & 109.43 & 0.450 & -0.436 & -0.702 & 2.350 & & \\
\hline B-12X-5, 109 & 109.61 & 0.322 & -0.434 & -0.879 & 2.513 & & \\
\hline B- $12 X-6,16$ & 109.88 & 0.394 & 0.059 & -0.734 & 2.523 & & \\
\hline B- $12 X-6,41$ & 110.13 & 0.162 & -0.116 & -0.815 & 2.613 & 18.0 & \\
\hline B- $12 X-6,66$ & 110.38 & 0.147 & 0.716 & -1.168 & 2.641 & & \\
\hline B-12X-6, 91 & 110.63 & 0.213 & -0.032 & -1.014 & 2.638 & & \\
\hline B-12X-6, 116 & 110.88 & 0.192 & 0.306 & -1.032 & 2.635 & & \\
\hline B-12X-6, 141 & 111.13 & 0.414 & 0.577 & -1.006 & 2.436 & & \\
\hline B- $12 X-7,16$ & 111.36 & -0.015 & 0.481 & -1.068 & 2.465 & & \\
\hline B- $12 X-7,41$ & 111.61 & 0.067 & 0.528 & -0.955 & 2.739 & & \\
\hline B- $12 X-7,51$ & 111.71 & 0.142 & 0.542 & -1.029 & 2.466 & & \\
\hline B- $13 X-1,16$ & 113.08 & 0.400 & 0.088 & -0.788 & 2.626 & & \\
\hline B-13X-1, 45 & 113.37 & 0.298 & 0.045 & -0.828 & 2.671 & & \\
\hline B-13X-1, 66 & 113.57 & 0.430 & 0.116 & -0.786 & 2.667 & & \\
\hline B-13X-1, 116 & 113.81 & 0.294 & 0 & -0.849 & 2.737 & & \\
\hline B-13X-1, 141 & 114.06 & 0.325 & 0.035 & -0.812 & 2.751 & & \\
\hline B- $13 X-2,11$ & 114.26 & 0.376 & -0.027 & -0.818 & 2.687 & & \\
\hline B- $13 X-2,41$ & 114.41 & 0.266 & -0.184 & -0.867 & 2.703 & & \\
\hline A- $13 \mathrm{X}-1,41$ & 114.51 & 0.080 & -0.161 & -0.691 & 2.764 & & \\
\hline B-13X-2, 90 & 114.60 & 0.326 & -0.159 & -0.910 & 2.404 & & \\
\hline B-13X-2, 116 & 114.86 & 0.229 & -0.795 & -0.850 & 2.625 & & \\
\hline B- $13 \mathrm{X}-2,141$ & 115.11 & 0.347 & -0.032 & -0.734 & 2.778 & & \\
\hline B- $13 X-3,5$ & 115.23 & 0.385 & -0.005 & -0.732 & 2.659 & & \\
\hline $\mathrm{A}-13 \mathrm{X}-1,116$ & 115.26 & 0.320 & -0.256 & -0.746 & 2.774 & & \\
\hline B-13X-3, 41 & 115.44 & 0.232 & -0.427 & -0.851 & 2.706 & & \\
\hline B- $13 X-3,66$ & 115.65 & 0.147 & -0.068 & -0.782 & 2.561 & & \\
\hline B-13X-3, 116 & 115.93 & 0.038 & 0.013 & -0.793 & 2.766 & & \\
\hline A- $13 X-2,41$ & 116.01 & 0.133 & -0.043 & -0.543 & 2.524 & & \\
\hline B-13X-3, 141 & 116.11 & 0.482 & 0.244 & -0.707 & 2.712 & & \\
\hline $\mathrm{A}-13 \mathrm{X}-2,126$ & 116.36 & 0.311 & -0.261 & -1.195 & 2.233 & & \\
\hline B- $13 X-4,16$ & 116.36 & 0.622 & 0.291 & -0.697 & 2.625 & & \\
\hline B- $13 X-4,43$ & 116.50 & 0.153 & -0.288 & -0.811 & 2.646 & & \\
\hline B-13X-4, 63 & 116.68 & 0.340 & -0.058 & -0.712 & 2.719 & & \\
\hline B-13X-4, 116 & 116.94 & 0.313 & 0.526 & -0.802 & 2.762 & & \\
\hline A- $13 \mathrm{X}-3,41$ & 117.01 & 0.281 & -0.342 & & & & \\
\hline B-13X-4, 141 & 117.18 & 0.334 & 0.159 & -0.812 & 2.592 & & \\
\hline
\end{tabular}


Table 1 (continued).

\begin{tabular}{|c|c|c|c|c|c|c|c|}
\hline \multirow{2}{*}{$\begin{array}{l}\text { Hole, core, section, } \\
\text { interval (cm) }\end{array}$} & \multirow{2}{*}{$\begin{array}{l}\text { Depth } \\
\text { (mbsf) }\end{array}$} & $\delta^{13} C_{P}$ & $\delta^{18} \mathrm{O}_{\mathrm{P}}$ & $\delta^{13} C_{B}$ & $\delta^{13} \mathrm{O}_{\mathrm{B}}$ & \multirow{2}{*}{$\begin{array}{l}\delta^{18} \mathrm{O} \\
\text { Stage }\end{array}$} & \multirow{2}{*}{$\begin{array}{l}\text { Nannofossil } \\
\text { datum }\end{array}$} \\
\hline & & \multicolumn{4}{|c|}{$\%_{0}$ PDB } & & \\
\hline B- $13 X-5,16$ & 117.41 & 0.240 & 0.015 & -0.652 & 2.661 & & \\
\hline B- $13 X-5,43$ & 117.65 & 0.465 & 0.406 & -0.841 & 2.698 & & \\
\hline A- $13 \mathrm{X}-3,116$ & 117.76 & 0.238 & -0.558 & & & & \\
\hline B- $13 X-5,66$ & 117.88 & 0.044 & -0.048 & -0.876 & 2.648 & & \\
\hline B- $13 X-5,116$ & 118.08 & & & -0.880 & 2.723 & & \\
\hline B-13X-5, 141 & 118.31 & 0.249 & -0.051 & -1.123 & 2.603 & & \\
\hline B- $13 X-6,16$ & 118.43 & 0.173 & 0.240 & -1.268 & 2.663 & & \\
\hline A-13X-4, 61 & 118.46 & 0.221 & -0.412 & -0.397 & 2.348 & & \\
\hline B- $13 X-6,43$ & 118.68 & 0.265 & -0.006 & -1.117 & 2.666 & & \\
\hline B- $13 X-6,57$ & 118.82 & 0 & 0.435 & -1.140 & 2.679 & & \\
\hline A- $13 X-5,41$ & 119.69 & 0.215 & -0.378 & -0.494 & 2.731 & 19.0 & \\
\hline B- $14 X-1,11$ & 122.69 & 0.184 & -0.016 & -0.583 & 2.654 & & \\
\hline B- $14 X-1,66$ & 122.97 & -0.024 & 0.095 & -0.621 & 2.835 & & \\
\hline B-14X-1, 91 & 123.22 & 0.284 & -0.180 & -0.664 & 2.670 & 20.0 & \\
\hline B- $14 X-1,116$ & 123.46 & 0.412 & 0.036 & -0.727 & 2.779 & 20.0 & \\
\hline B-14X-1, 141 & 123.71 & 0.471 & 0.196 & -0.738 & 2.725 & & \\
\hline B-14X-2, 11 & 123.88 & 0.466 & 0.292 & -0.750 & 2.653 & & \\
\hline B-14X-2, 71 & 124.15 & 0.416 & 0.031 & -0.533 & 2.656 & & \\
\hline B-14X-2, 91 & 124.35 & 0.419 & 0.292 & -0.652 & 2.675 & & \\
\hline B-14X-2, 116 & 124.57 & 0.255 & 0.303 & -0.698 & 2.554 & & \\
\hline A- $14 X-1,147$ & 124.72 & 0.304 & -0.181 & -0.604 & 2.453 & & \\
\hline B-14X-3, 11 & 124.81 & 0.451 & 0.194 & -0.696 & 2.551 & & \\
\hline B-14X-3, 41 & 125.09 & 0.424 & 0.183 & -0.685 & 2.564 & & \\
\hline B-14X-3, 69 & 125.37 & 0.486 & -0.177 & -0.586 & 2.520 & & \\
\hline B-14X-3, 91 & 125.59 & 0.491 & 0.048 & -0.650 & 2.602 & & \\
\hline B- $14 X-3,116$ & 125.84 & 0.550 & -0.060 & -0.531 & 2.676 & & \\
\hline A-14X-2, 129 & 125.94 & -0.034 & -0.240 & -0.663 & 2.333 & & \\
\hline B-14X-3, 139 & 126.07 & 0.414 & 0 & -0.581 & 2.720 & & \\
\hline B- $14 X-4,11$ & 126.17 & 0.432 & -0.153 & -0.633 & 2.634 & & \\
\hline B- $14 X-4,64$ & 126.45 & 0.250 & -0.114 & -0.575 & 2.550 & & \\
\hline B- $14 X-4,91$ & 126.72 & 0.638 & -0.039 & -0.488 & 2.565 & & \\
\hline A-14X-3, 116 & 126.94 & 0.144 & -0.297 & -0.487 & 2.596 & & \\
\hline B- $14 X-4,116$ & 126.95 & 0.588 & -0.240 & -0.471 & 2.607 & & \\
\hline B- $14 X-4,141$ & 127.20 & 0.457 & 0.007 & -0.453 & 2.513 & 210 & \\
\hline B-14X-5, 11 & 127.40 & 0.301 & -0.196 & -0.494 & 2.492 & 21.0 & \\
\hline B-14X-5, 31 & 127.60 & 0.465 & -0.225 & -0.594 & 2.540 & & \\
\hline B-14X-5, 71 & 127.95 & 0.571 & -0.101 & -0.665 & 2.556 & & \\
\hline A-14X-4, 109 & 128.07 & 0.256 & -0.331 & -0.667 & 2.267 & & \\
\hline B-14X-5, 91 & 128.14 & 0.340 & -0.550 & -0.654 & 2.362 & & \\
\hline B- $14 X-5,116$ & 128.34 & 0.287 & -0.240 & -0.806 & 2.178 & & \\
\hline B-14X-5, 141 & 128.59 & 0.457 & -0.425 & -0.659 & 2.235 & & \\
\hline B- $14 X-6,11$ & 128.79 & 0.415 & -0.394 & -0.585 & 2.238 & & \\
\hline B-14X-6, 41 & 129.09 & 0.291 & -0.338 & -0.466 & 2.428 & & \\
\hline B- $14 X-6,66$ & 129.32 & 0.282 & -0.287 & -0.558 & 2.271 & & \\
\hline B-14X-6, 91 & 129.57 & 0.377 & -0.495 & -0.617 & 2.161 & & \\
\hline B- $14 X-6,116$ & 129.82 & 0.136 & -0.536 & -0.640 & 2.357 & & \\
\hline B-14X-6, 141 & 130.07 & 0.430 & -0.310 & -0.508 & 2.320 & & \\
\hline B-15X-7, 11 & 130.27 & 0.195 & -0.289 & -0.575 & 2.272 & & \\
\hline B- $15 X-1,16$ & 132.46 & -0.299 & 0.303 & -0.939 & 2.523 & 22.0 & \\
\hline B-15X-1, 41 & 132.71 & -0.204 & 0.638 & -1.397 & 2.715 & & \\
\hline B-15X-1, 64 & 132.94 & 0.126 & 0.318 & -0.933 & 2.629 & & \\
\hline B-15X-1, 88 & 133.18 & -0.049 & 0.378 & -1.170 & 2.691 & & \\
\hline B- $15 X-1,116$ & 133.46 & -0.285 & 0.587 & -1.001 & 2.570 & & \\
\hline B- $15 X-1,141$ & 133.71 & 0.078 & 0.591 & -1.315 & 2.734 & & \\
\hline B- $15 X-2,16$ & 133.96 & -0.080 & 0.625 & -1.103 & 2.747 & & \\
\hline B- $15 X-2,38$ & 134.18 & & & -1.264 & 2.885 & & \\
\hline B- $15 X-2,69$ & 134.49 & -0.120 & 0.405 & -1.230 & 2.897 & & \\
\hline B-15X-2, 91 & 134.71 & -0.086 & 0.596 & -1.234 & 2.769 & & \\
\hline B-15X-2, 138 & 134.88 & 0.082 & 0.622 & -1.113 & 2.740 & & \\
\hline B- $15 X-3,8$ & 135.05 & -0.015 & 0.629 & -1.254 & 2.854 & & \\
\hline B- $15 X-3,38$ & 135.25 & -0.159 & 0.662 & -1.265 & 2.735 & & \\
\hline B-15X-3, 63 & 135.50 & -0.139 & 0.701 & -1.047 & 2.619 & & \\
\hline B- $15 X-3,116$ & 135.73 & 0.042 & 0.613 & -1.015 & 2.524 & & \\
\hline B- $15 X-3,141$ & 135.98 & 0.002 & 0.555 & -1.060 & 2.663 & & \\
\hline A- $15 X-3,146$ & 136.20 & -0.005 & 0.582 & -1.145 & 2.694 & & \\
\hline B- $15 X-4,16$ & 136.21 & -0.031 & 0.679 & -0.973 & 2.559 & & \\
\hline B- $15 X-4,43$ & 136.39 & -0.211 & 0.652 & -0.961 & 2.560 & & \\
\hline B- $15 X-4,69$ & 136.65 & -0.003 & 0.714 & -1.035 & 2.178 & & \\
\hline B- $15 X-4,96$ & 136.77 & -0.025 & 0.614 & -1.032 & 2.333 & & \\
\hline B- $15 X-4,116$ & 136.97 & -0.022 & 0.677 & -0.973 & 2.529 & & \\
\hline B- $15 X-4,141$ & 137.20 & -0.022 & 0.725 & -1.121 & 2.481 & & \\
\hline B- $15 X-5,16$ & 137.45 & -0.093 & 0.607 & -1.014 & 2.603 & & \\
\hline B-15X-5, 45 & 137.73 & -0.039 & 0.687 & -0.936 & 2.652 & & \\
\hline B- $15 X-5,69$ & 137.97 & -0.073 & 0.495 & -1.006 & 2.706 & & \\
\hline A-15X-5, 119 & 138.16 & -0.111 & 0.027 & -0.955 & 2.620 & & \\
\hline B- $15 X-5,91$ & 138.19 & -0.127 & 0.621 & -1.066 & 2.738 & & \\
\hline B- $15 X-5,116$ & 138.42 & -0.299 & 0.908 & -1.001 & 2.625 & & \\
\hline
\end{tabular}


Table 1 (continued).

\begin{tabular}{|c|c|c|c|c|c|c|c|}
\hline \multirow{2}{*}{$\begin{array}{l}\text { Hole, core, section, } \\
\text { interval }(\mathrm{cm})\end{array}$} & \multirow{2}{*}{$\begin{array}{l}\text { Depth } \\
\text { (mbsf) }\end{array}$} & $\delta^{13} \mathrm{C}_{\mathrm{P}}$ & $\delta^{18} \mathrm{O}_{\mathrm{P}}$ & $\delta^{13} C_{B}$ & $\delta^{13} \mathrm{O}_{\mathrm{B}}$ & \multirow{2}{*}{$\begin{array}{l}\delta^{18} \mathrm{O} \\
\text { Stage }\end{array}$} & \multirow{2}{*}{$\begin{array}{c}\text { Nannofossil } \\
\text { datum }\end{array}$} \\
\hline & & \multicolumn{4}{|c|}{$\%_{0}$ PDB } & & \\
\hline B- $15 X-5,141$ & 138.67 & -0.054 & 0.571 & -1.049 & 2.672 & & \\
\hline B- $15 X-6,16$ & 138.92 & -0.190 & 0.507 & -1.092 & 2.716 & & \\
\hline B- $15 X-6,42$ & 139.17 & -0.182 & 0.553 & -0.870 & 2.696 & & \\
\hline A- $15 X-6,117$ & 139.30 & -0.013 & -0.044 & -1.049 & 2.569 & & \\
\hline B- $15 X-6,67$ & 139.42 & -0.273 & 1.336 & -1.059 & 2.744 & 230 & \\
\hline B- $15 X-6,92$ & 139.67 & -0.232 & 0.142 & -1.033 & 2.736 & 23.0 & \\
\hline B- $15 X-6,116$ & 139.91 & 0.001 & 0.117 & -1.218 & 2.634 & & \\
\hline B-15X-6, 141 & 140.16 & -0.109 & 0.116 & & & & \\
\hline B- $16 X-1,15$ & 142.05 & -0.100 & 0.385 & -1.229 & 2.569 & & \\
\hline B-16X-1, 145 & 143.00 & -0.181 & 0.177 & -0.998 & 2.742 & & \\
\hline B- $16 X-2,15$ & 143.20 & & & -0.875 & 2.739 & & TOP Acm. \\
\hline B- $16 X-2,116$ & 143.58 & 0.183 & 0.450 & -0.990 & 2.653 & & $R . s p$. A \\
\hline B- $16 X-3,15$ & 144.02 & -0.098 & -0.041 & -0.686 & 3.109 & & \\
\hline B- $16 X-3,116$ & 145.01 & -0.020 & 0.234 & -0.968 & 2.544 & 24 & \\
\hline B-17X-1, 41 & 149.31 & 0.167 & -0.488 & -0.892 & 2.549 & 24 & \\
\hline B-17X-1, 116 & 150.06 & 0.464 & -0.122 & -0.804 & 2.324 & & \\
\hline B-17X-2, 51 & 150.86 & 0.521 & -0.762 & -0.594 & 2.376 & & \\
\hline B-17X-2, 133 & 151.65 & 0.619 & -0.793 & -0.590 & 2.292 & 25 & \\
\hline B-17X-3, 67 & 152.17 & 0.422 & -0.610 & -0.509 & 2.225 & & \\
\hline B-17X-3, 113 & 152.61 & & & -0.509 & 2.311 & & \\
\hline B- $17 X-4,41$ & 153.37 & 0.344 & -0.340 & -0.649 & 2.282 & & \\
\hline A-17X-1, 102 & 153.77 & 0.505 & -0.376 & -0.538 & 2.286 & & \\
\hline B- $17 X-4,119$ & 154.15 & 0.039 & -0.350 & -0.704 & 2.520 & & \\
\hline B-17X-5, 41 & 154.78 & 0.206 & -0.274 & -0.670 & 2.465 & & \\
\hline A-17X-2, 110 & 155.25 & 0.412 & 0.032 & -0.717 & 2.468 & 26 & \\
\hline B-17X-5, 116 & 155.46 & 0.355 & -0.078 & -0.826 & 2.626 & & \\
\hline B-17X-6, 41 & 156.21 & 0.161 & -0.641 & -0.852 & 2.700 & 27 & \\
\hline B- $17 X-6,116$ & 156.96 & 0.138 & -0.222 & -0.802 & 2.441 & & \\
\hline B- $17 \mathrm{X}-\mathrm{CC}, 41$ & 157.39 & 0.193 & -0.052 & -0.794 & 2.535 & & 1 \\
\hline B- $18 X-1,41$ & 159.01 & 0.109 & -0.311 & -0.750 & 2.349 & & \\
\hline B- $18 X-1,121$ & 159.81 & 0.011 & -0.431 & -0.814 & 2.627 & & \\
\hline B-18X-2, 53 & 160.53 & -0.059 & 0.213 & -0.781 & 2.595 & 28 & \\
\hline B- $18 X-2,121$ & 161.01 & -0.073 & 0.041 & & & & \\
\hline B- $18 X-3,41$ & 161.71 & 0.040 & 0.055 & & & & \\
\hline B- $18 X-3,124$ & 162.38 & -0.174 & -0.094 & -0.844 & 2.424 & & LAD \\
\hline B- $18 X-4,41$ & 163.01 & 0.283 & 0.048 & -0.891 & 2.724 & & G. parallela \\
\hline B- $18 X-4,125$ & 163.48 & 0.111 & -0.063 & -0.644 & 3.105 & & - $10-5$ \\
\hline B-18X-5, 54 & 164.01 & 0.104 & -0.086 & -0.798 & 2.519 & & \\
\hline B-18X-5, 121 & 164.66 & 0.284 & -0.016 & -0.768 & 2.552 & & \\
\hline B- $18 X-6,41$ & 165.24 & & & -0.661 & 2.584 & & \\
\hline B- $18 X-6,121$ & 166.04 & 0.235 & -0.352 & -0.696 & 2.310 & 29 & \\
\hline B- $18 X-7,24$ & 166.57 & 0.384 & -0.263 & -0.937 & 2.356 & & \\
\hline A-19X-1, 116 & 173.26 & 0.567 & -0.060 & -0.788 & 2.370 & & \\
\hline A-19X-2, 116 & 174.42 & -0.071 & -0.049 & & & & \\
\hline A-19X-3, 116 & 175.61 & -0.286 & -0.120 & -0.901 & 2.922 & & \\
\hline A- $19 X-4,104$ & 176.70 & 0.118 & 0.221 & & & & \\
\hline A-19X-5, 112 & 117.75 & 0.031 & 0.256 & & & 30 & \\
\hline A-19X-6, 116 & 178.76 & 0.177 & -0.029 & & & & \\
\hline A- $19 X-7,116$ & 180.25 & 0.159 & -0.370 & -0.612 & 2.211 & & \\
\hline A- $20 \mathrm{X}-1,36$ & 182.16 & 0.055 & -0.638 & -0.619 & 2.076 & 31 & \\
\hline A- $20 \mathrm{X}-1,132$ & 182.79 & 0.239 & -0.180 & -0.822 & 2.310 & & \\
\hline A-20X-2, 51 & 183.23 & 0.134 & -0.326 & -0.681 & 2.245 & & \\
\hline A-20X-2, 139 & 183.81 & 0.245 & -0.341 & -0.717 & 2.329 & & \\
\hline A- $20 X-3,36$ & 183.96 & 0.063 & -0.240 & -0.659 & 2.366 & & \\
\hline A-20X-3, 138 & 184.74 & 0.203 & -0.181 & -0.844 & 2.490 & 32 & \\
\hline A- $20 \times-4,36$ & 185.12 & 0.097 & -0.806 & -0.579 & 2.506 & 33 & \\
\hline A- $20 X-4,145$ & 185.74 & 0.054 & -0.379 & -0.761 & 2.401 & & \\
\hline A-20X-5, 36 & 186.03 & 0.047 & 0.018 & & & & \\
\hline A- $20 X-5,133$ & 186.66 & 0.138 & -0.274 & -0.875 & 2.664 & & \\
\hline A- $20 X-6,46$ & 187.08 & 0.065 & -0.166 & -0.941 & 2.683 & & \\
\hline A- $20 \times-6,127$ & 187.69 & 0.023 & 0.22 & -0.813 & 2.513 & 34 & \\
\hline A-20X-7, 36 & 188.08 & 0.106 & -0.213 & -0.840 & 2.389 & & 4 LAD \\
\hline A- 21 X-2, 139 & 193.06 & -0.097 & -0.280 & -0.906 & 2.207 & 35 & G. (large) \\
\hline A- $21 X-3,114$ & 193.70 & -0.233 & 0.127 & -1.263 & 2.175 & & \\
\hline A- $21 X-4,103$ & 194.54 & 0.142 & 0.279 & -0.623 & 2.467 & & \\
\hline A-21X-5, 134 & 195.55 & 0.429 & 0.361 & -0.666 & 2.424 & 36 & \\
\hline A-22X-1, 41 & 201.51 & 0.103 & -0.188 & & & & \\
\hline A-22X-1, 118 & 201.98 & 0.280 & -0.058 & -0.550 & 2.461 & 37 & \\
\hline A- $22 X-2,32$ & 202.55 & -0.162 & -0.557 & -0.523 & 2.335 & 37 & \\
\hline A- $22 X-2,116$ & 202.77 & -0.192 & 0.136 & -0.595 & 2.352 & & \\
\hline A- $22 \mathrm{X}-3,32$ & 203.36 & -0.066 & -0.305 & -0.838 & 2.344 & & \\
\hline A- $22 X-3,144$ & 204.00 & -0.127 & -0.393 & -0.601 & 2.118 & & \\
\hline A- $22 X-4,41$ & 204.36 & -0.461 & 0.418 & -0.740 & 2.287 & 38 & \\
\hline A- $22 X-4,137$ & 204.88 & 0.012 & -0.225 & -0.896 & 2.311 & & \\
\hline A- $22 X-5,41$ & 205.14 & -0.079 & -0.134 & -1.175 & 1.767 & & \\
\hline A-22X-5, 141 & 205.84 & 0.003 & -0.067 & -0.482 & 1.813 & & \\
\hline A- $22 X-6,26$ & 206.19 & -0.198 & -0.065 & -0.625 & 2.593 & & \\
\hline
\end{tabular}


Table 1 (continued).

\begin{tabular}{|c|c|c|c|c|c|c|c|}
\hline \multirow{2}{*}{$\begin{array}{l}\text { Hole, core, section, } \\
\text { interval }(\mathrm{cm})\end{array}$} & \multirow{2}{*}{$\begin{array}{l}\text { Depth } \\
\text { (mbsf) }\end{array}$} & $\delta^{13} C_{P}$ & $\delta^{18} \mathrm{O}_{\mathrm{P}}$ & $\delta^{13} \mathrm{C}_{\mathrm{B}}$ & $\delta^{13} \mathrm{O}_{\mathrm{B}}$ & \multirow{2}{*}{$\begin{array}{l}\delta^{18} \mathrm{O} \\
\text { Stage }\end{array}$} & \multirow{2}{*}{$\begin{array}{l}\text { Nannofossil } \\
\text { datum }\end{array}$} \\
\hline & & \multicolumn{4}{|c|}{$\%_{0}$ PDB } & & \\
\hline A-22X-6, 116 & 206.84 & & & -0.565 & 2.536 & & \\
\hline B-23X-1, 41 & 207.31 & -0.154 & -0.949 & -0.873 & 2.177 & & \\
\hline A-22X-7, 40 & 207.55 & 0.405 & -0.132 & -0.642 & 2.561 & & \\
\hline B- $23 X-2,41$ & 207.73 & -0.089 & -0.429 & -0.899 & 2.027 & 39 & \\
\hline A- $22 \mathrm{X}-\mathrm{CC}, 40$ & 207.98 & & & -0.598 & 2.339 & & \\
\hline B-23X-3, 41 & 209.22 & & & -0.925 & 2.500 & & \\
\hline B-23X-3, 124 & 210.03 & & & -0.832 & 2.731 & & \\
\hline B- $23 X-4,41$ & 210.60 & -0.021 & -0.420 & -0.726 & 2.759 & & \\
\hline B- $23 \times-4,119$ & 211.31 & 0.244 & -0.187 & -0.741 & 2.506 & & \\
\hline A- $23 X-2,56$ & 211.96 & & & -0.600 & 2.945 & & \\
\hline A- $23 X-2,26$ & 212.31 & & & -0.588 & 3.020 & & \\
\hline A- $23 \mathrm{X}-3,32$ & 213.13 & 0.185 & -0.246 & -0.691 & 2.927 & & \\
\hline B-23X-6, 41 & 213.14 & 0.359 & -0.131 & -0.380 & 2.538 & & \\
\hline B-23X-6, 93 & 213.66 & & & -0.640 & 2.409 & & \\
\hline B-23X-7, 36 & 214.34 & 0.06 & -0.263 & & & & \\
\hline B-24X-1, 139 & 217.74 & -0.035 & 0.045 & & & 40 & \\
\hline B-24X-2, 105 & 218.90 & & & -0.629 & 2.342 & & \\
\hline B- $24 X-3,39$ & 219.37 & & & -0.557 & 2.436 & & \\
\hline B- $24 X-3,101$ & 219.84 & & & -0.455 & 2.365 & & \\
\hline B- $24 X-4,41$ & 220.54 & & & -0.625 & 2.183 & & \\
\hline A-24X-1, 41 & 220.81 & & & -0.600 & 2.466 & & \\
\hline A-24X-1, 116 & 221.56 & & & -0.709 & 2.299 & & \\
\hline B-24X-5, 35 & 221.95 & 0.476 & -0.735 & -0.601 & 2.018 & & \\
\hline A- $24 X-2,61$ & 220.08 & & & -0.712 & 2.104 & & \\
\hline A- $24 X-2,116$ & 222.63 & & & -0.746 & 2.015 & & \\
\hline B- $24 X-6,35$ & 222.68 & -0.028 & -0.831 & & & & \\
\hline B- $24 X-6,125$ & 223.08 & -0.073 & -0.700 & & & 41 & 1 \\
\hline B- $24 X-7,36$ & 223.65 & 0.023 & -0.048 & -0.448 & 2.426 & & \\
\hline A- $24 X-3,127$ & 223.95 & & & -0.678 & 2.325 & & \\
\hline A- $24 X-4,35$ & 224.45 & 0.030 & -0.548 & -0.817 & 2.408 & & \\
\hline A- $24 X-4,93$ & 224.73 & -0.284 & -0.757 & -0.705 & 2.363 & & . \\
\hline A- $24 X-5,41$ & 225.33 & 0.002 & 0.158 & & & & LAD \\
\hline A-24X-5, 113 & 226.05 & -0.081 & 0.155 & -0.540 & 2.588 & & H. sellii \\
\hline A-24X-6, 43 & 226.51 & 0.091 & 0.216 & -0.683 & 2.673 & & \\
\hline A- $24 X-6,116$ & 227.24 & & & -0.370 & 2.553 & & \\
\hline B-25X-1, 146 & 227.26 & & & -0.316 & 2.227 & 42 & \\
\hline A-24X-7, 33 & 227.83 & 0.557 & -0.115 & -0.407 & 2.351 & & \\
\hline B- $25 X-2,140$ & 228.24 & 0.282 & -0.239 & -0.317 & 2.529 & & \\
\hline A- $24 X-C C, 41$ & 228.28 & & & -0.524 & 2.377 & & \\
\hline B-25X-3, 116 & 229.38 & 0.474 & -0.700 & -0.222 & 2.661 & 43 & \\
\hline A-25X-1, 74 & 230.39 & 0.144 & 0.264 & -0.284 & 2.617 & 44 & \\
\hline B-25X-4, 118 & 230.57 & & & -0.139 & 2.685 & & \\
\hline A- $25 X-2,73$ & 230.83 & 0.028 & -0.048 & -0.330 & 2.813 & & \\
\hline A- $25 X-2,111$ & 231.19 & 0.305 & -0.030 & -0.350 & 2.741 & 45 & \\
\hline B- $25 X-5,114$ & 231.38 & & & -0.143 & 2.416 & & \\
\hline A- $25 X-3,9$ & 231.51 & 0.240 & -0.026 & -0.395 & 2.746 & & \\
\hline A-25X-4, 68 & 232.02 & 0.415 & 0.148 & -0.308 & 2.685 & & \\
\hline A- $25 X-4,111$ & 232.25 & & & -0.306 & 2.585 & & \\
\hline B- $25 X-6,106$ & 232.30 & & & -0.518 & 2.415 & & \\
\hline A- $25 X-5,68$ & 232.68 & & & -0.313 & 2.634 & & \\
\hline B- $25 X-7,22$ & 232.76 & & & -0.675 & 2.554 & & \\
\hline A- $25 X-5,111$ & 232.84 & & & -0.325 & 2.459 & & \\
\hline A- $25 X-6,55$ & 233.78 & 0.208 & 0.437 & -0.616 & 2.674 & 46 & \\
\hline A- $25 X-6,110$ & 234.25 & 0.071 & -0.037 & -0.415 & 2.677 & & \\
\hline A- $25 X-7,20$ & 234.80 & & & -0.601 & 2.832 & & \\
\hline B-26X-1, 129 & 236.99 & & & -0.720 & 2.485 & & \\
\hline B-26X-2, 112 & 238.32 & & & -0.598 & 2.566 & & \\
\hline B-26X-3, 44 & 238.63 & 0.308 & -0.329 & -0.648 & 2.369 & 47 & \\
\hline B- $26 \mathrm{X}-3,120$ & 239.39 & & & -0.752 & 2.373 & & \\
\hline B- $26 \mathrm{X}-4,31$ & 239.87 & & & -0.729 & 2.300 & & \\
\hline A-26X-1, 40 & 240.20 & & & -0.617 & 2.563 & & \\
\hline B- $26 \mathrm{X}-4,111$ & 240.39 & & & -0.694 & 2.253 & & \\
\hline A-26X-2, 33 & 240.94 & & & -0.631 & 2.426 & & \\
\hline A-26X-3, 30 & 241.66 & & & -0.711 & 2.333 & & \\
\hline A- $26 \mathrm{X}-3,90$ & 242.01 & & & -0.775 & 2.263 & & \\
\hline A-26X-4, 33 & 242.79 & & & -0.727 & 2.306 & & \\
\hline A- $26 \mathrm{X}-4,115$ & 243.53 & 0.419 & 0.147 & -0.439 & 2.427 & & \\
\hline A-26X-5, 3 & 243.71 & & & -0.475 & 2.731 & & LAD \\
\hline A- $27 \mathrm{X}-1,40$ & 249.80 & & & -0.475 & 2.731 & & 1C. macintyrei \\
\hline A- $27 X-2,40$ & 250.70 & & & -0.603 & 2.792 & & \\
\hline A-27X-2, 125 & 251.38 & 0.512 & -0.035 & -0.510 & 2.761 & & \\
\hline A-27X-3, 40 & 251.88 & & & -0.449 & 2.767 & & \\
\hline A-27X-3, 129 & 252.29 & 0.167 & 0.194 & -0.514 & 2.613 & & \\
\hline A- $27 X-4,40$ & 252.90 & & & -0.535 & 2.684 & & \\
\hline A- $27 X-4,120$ & 253.55 & & & -0.487 & 2.663 & & \\
\hline A-27X-6, 40 & 255.43 & -0.068 & 0.085 & & & & \\
\hline A-28X-1, 115 & 260.17 & 0.067 & -0.128 & -0.648 & 2.354 & & \\
\hline
\end{tabular}


Table 1 (continued).

\begin{tabular}{|c|c|c|c|c|c|c|c|}
\hline \multirow{2}{*}{$\begin{array}{l}\text { Hole, core, section, } \\
\text { interval }(\mathrm{cm})\end{array}$} & \multirow{2}{*}{$\begin{array}{l}\text { Depth } \\
\text { (mbsf) }\end{array}$} & $\delta^{13} \mathrm{C}_{\mathrm{P}}$ & $\delta^{18} \mathrm{O}_{\mathrm{P}}$ & $\delta^{13} \mathrm{C}_{\mathrm{B}}$ & $\delta^{13} \mathrm{O}_{\mathrm{B}}$ & \multirow{2}{*}{$\begin{array}{l}\delta^{18} \mathrm{O} \\
\text { Stage }\end{array}$} & \multirow{2}{*}{$\begin{array}{l}\text { Nannofossil } \\
\text { datum }\end{array}$} \\
\hline & & \multicolumn{4}{|c|}{$\%_{0}$ PDB } & & \\
\hline A- $29 X-1,40$ & 269.10 & -0.194 & 0.271 & -0.724 & 2.404 & & \\
\hline A-29X-1, 113 & 269.51 & & & -0.924 & 2.277 & & \\
\hline A- $29 X-2,40$ & 269.98 & & & -0.697 & 2.491 & & \\
\hline A- $29 X-3,40$ & 270.77 & & & -0.521 & 2.632 & & \\
\hline A-29X-3, 115 & 271.24 & -0.189 & -0.229 & -0.688 & 2.328 & & \\
\hline A- $29 X-4,40$ & 271.91 & 0.064 & -0.485 & -0.866 & 2.272 & & \\
\hline A- $29 X-4,108$ & 272.38 & 0.037 & -0.575 & -0.766 & 2.472 & & \\
\hline A- $29 X-5,40$ & 273.08 & -0.376 & -0.750 & & & $53 ?$ & \\
\hline A-29X-5, 117 & 273.62 & -0.116 & -0.168 & -0.759 & 2.303 & & \\
\hline A- $29 X-6,40$ & 274.18 & 0.032 & -0.219 & -1.013 & 2.522 & & \\
\hline A-29X-6, 106 & 274.84 & 0.032 & -0.178 & & & & \\
\hline A-30X-1, 88 & 279.18 & 0.191 & -0.436 & -0.541 & 2.802 & A & \\
\hline A-30X-4, 109 & 280.84 & & & -0.555 & 2.637 & & FAD \\
\hline A-31X-3, 137 & 290.43 & 0.103 & -0.227 & -0.731 & 2.394 & & G. carribianica \\
\hline A- $31 X-4,125$ & 291.31 & & & -0.386 & 2.640 & & \\
\hline B-31X-1, 81 & 294.51 & 0.229 & -0.603 & & & & \\
\hline B- $31 X-2,86$ & 295.76 & & & -0.441 & 2.563 & & \\
\hline B- $31 X-3,89$ & 296.12 & & & -0.457 & 2.552 & & \\
\hline B- $31 \times-4,92$ & 297.03 & & & -0.567 & 2.535 & & \\
\hline B-31X-5, 114 & 298.05 & & & -0.467 & 2.190 & & \\
\hline $\mathrm{A}-32 \mathrm{X}-1,116$ & 298.66 & & & -0.381 & 2.334 & & \\
\hline A-32X-2, 129 & 299.76 & & & -0.339 & 2.371 & & \\
\hline A- $32 X-4,100$ & 302.27 & & & -0.376 & 2.364 & & \\
\hline A-32X-6, 95 & 304.93 & & & -0.373 & 2.527 & & \\
\hline A-33X-2, 130 & 308.63 & & & -0.620 & 2.649 & & \\
\hline A- $33 \mathrm{X}-3,105$ & 309.83 & & & -0.571 & 2.606 & & \\
\hline A- 33 X- 6,105 & 313.80 & -0.276 & -0.117 & -0.500 & 2.319 & & \\
\hline B- $33 X-3,59$ & 315.61 & & & -0.538 & 2.269 & & \\
\hline A- $34 \mathrm{X}-1,18$ & 316.88 & & & -0.684 & 2.501 & & \\
\hline A- $35 \mathrm{X}-1,40$ & 321.90 & & & -0.443 & 2.387 & & \\
\hline A-35X-1, 126 & 322.53 & & & -0.415 & 2.433 & & \\
\hline A-35X-2, 42 & 323.01 & & & -0.480 & 2.326 & & \\
\hline A-35X-2, 104 & 323.18 & & & -0.457 & 2.287 & & \\
\hline B- $35 X-1,116$ & 323.81 & & & -0.536 & 2.225 & & \\
\hline A-35X-3, 105 & 324.32 & & & -0.396 & 2.197 & & \\
\hline B- $35 X-2,120$ & 324.71 & & & -0.655 & 2.514 & & \\
\hline$A-35 X-4,53$ & 324.92 & & & -0.494 & 2.231 & & \\
\hline A- $35 X-4,94$ & 325.33 & & & -0.544 & 2.564 & & \\
\hline A-36X-1, 120 & 327.50 & & & -0.947 & 2.511 & & \\
\hline A-36X-2, 39 & 327.89 & & & -0.540 & 2.679 & A & \\
\hline A- $36 \mathrm{X}-4,108$ & 330.54 & & & -0.766 & 2.135 & 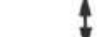 & LAD \\
\hline A-36X-6, 37 & 332.50 & & & -0.695 & 2.430 & & D. brouweri \\
\hline A- $36 X-7,37$ & 333.75 & & & -0.769 & 2.383 & & \\
\hline A-36X-7, 116 & 334.51 & & & -0.843 & 2.654 & & \\
\hline B-38X-1, 74 & 352.64 & & & -0.243 & 2.495 & & \\
\hline B- $38 X-2,121$ & 353.63 & & & -0.377 & 2.572 & & \\
\hline A- 39 X-1, 40 & 355.40 & & & -0.430 & 2.553 & & \\
\hline A-39X-1, 142 & 355.77 & & & -0.324 & 2.408 & & \\
\hline A-39X-2, 43 & 356.11 & & & -0.324 & 2.541 & & \\
\hline A-39X-2, 142 & 356.67 & & & -0.416 & 2.822 & & \\
\hline A- $39 X-3,43$ & 357.00 & & & -0.442 & 2.762 & & \\
\hline A- $39 \mathrm{X}-3,84$ & 357.21 & 0.113 & -0.279 & -0.411 & 2.695 & & \\
\hline A-39X-4, 41 & 357.55 & 0.016 & -0.355 & -0.438 & 2.664 & & \\
\hline A- $39 X-4,120$ & 358.27 & & & -0.501 & 2.647 & & \\
\hline A- 39 X-5, 41 & 358.79 & & & -0.588 & 2.318 & & \\
\hline A-39X-5, 112 & 359.15 & & & -0.587 & 2.230 & & \\
\hline A- $40 \mathrm{X}-1,72$ & 365.02 & -0.602 & -0.332 & & & & \\
\hline A $-40 X-1,131$ & 365.58 & & & -0.762 & 2.670 & & \\
\hline A- $40 X-2,76$ & 365.78 & & & -0.941 & 2.748 & & \\
\hline A- $-40 X-2,131$ & 366.20 & -0.505 & -0.198 & -0.759 & 2.683 & & \\
\hline A- $40 X-3,67$ & 366.39 & -0.583 & -0.022 & -0.649 & 2.584 & & \\
\hline A- $-40 X-4,70$ & 367.26 & & & -0.680 & 2.745 & & \\
\hline A- $40 X-5,40$ & 367.83 & & & -0.571 & 2.420 & & \\
\hline A- $-40 X-6,48$ & 369.16 & & & -0.783 & 2.460 & & \\
\hline A $-40 X-6,128$ & 369.94 & & & -0.737 & 2.464 & & \\
\hline A $-40 \times-7,33$ & 370.49 & & & -0.791 & 2.654 & & \\
\hline A- $40 \mathrm{X}-\mathrm{CC}, 30$ & 370.86 & & & -0.496 & 2.654 & & \\
\hline A- $41 X-3,71$ & 376.20 & & & -0.992 & 2.415 & & \\
\hline A- $41 X-4,12$ & 376.75 & & & -0.604 & 2.656 & & \\
\hline$A-41 \times-6,55$ & 378.89 & & & -0.657 & 2.357 & & \\
\hline A- $41 X-C C, 40$ & 379.37 & -0.380 & -0.349 & -0.803 & 2.523 & & \\
\hline B- $41 X-1,41$ & 381.41 & & & -0.807 & 2.484 & & \\
\hline
\end{tabular}


transgressive stages means that during the lightening of isotopic ratio of surface water mass (amount of global ice sheet reduced), the bottom temperature was colder than glacial stage or the lightening of isotopic ratio of bottom water mass was delayed. The delays are counted for depth $50-80 \mathrm{~cm}$ whose corresponding duration is calculated to be $2500-4000$ yr. The delays result in significant peaks of the difference of oxygen isotope ratio $\delta^{18} \mathrm{O}_{\mathrm{B}-\mathrm{P}}$ between benthic $\delta^{18} \mathrm{O}_{\mathrm{B}}$ and planktonic $\delta^{18} \mathrm{O}_{\mathrm{P}}$ in the transgressive stages.

\section{CARBON ISOTOPE STRATIGRAPHY}

The carbon isotope values range from $-0.6 \%$ to $0.7 \%$ for planktonic $\delta^{13} \mathrm{C}_{\mathrm{P}}$ and from $-1.7 \%$ to $-0.1 \%$ for benthic $\delta^{13} \mathrm{C}_{\mathrm{B}}$ (Fig. 3, Table 1). The heavier values in planktonic $\delta^{13} C_{\mathrm{P}}$ are consistent with the uptake of lighter carbon by the biological productivity in the surface water. The fluctuations of carbon isotope values for Stages 1-15 are 1.3\%o for benthic and planktonic foraminifers, $\delta^{13} C_{B}$, and $\delta^{13} C_{P}$ (Fig. 4), the changes and fluctuations of carbon isotopes are similar between benthic and planktonic. Generally $\delta^{13} C_{B}$ and $\delta^{13} C_{p}$ have an inverse correlation with oxygen isotope values $\delta^{18} \mathrm{O}_{\mathrm{B}}$ and $\delta^{18} \mathrm{O}_{\mathrm{P}}$, however, the changes of $\delta^{18} \mathrm{O}$ and $\delta^{13} \mathrm{C}$ are different. While the $\delta^{18} \mathrm{O}$ during transgressive stages decreases sharply, called "termination" (Broecker and vanDonk, 1970). The $\delta^{13} \mathrm{C}$ increases gradually, in spite of the synchronized changes of $\delta^{13} \mathrm{C}$ with that of $\delta^{18} \mathrm{O}$ during regressive stages; a typical case can be found during the transgressive stages from Stages 12-11, 10-9, 8-7, and 6-5.

$\delta^{18} \mathrm{O}$ of the seawater is controlled by the global ice volume correlated with eustatic sea level, and $\delta^{13} \mathrm{C}$ is influenced by the degree of the biological uptake of light carbon from the dissolved $\mathrm{\Sigma CO}_{2}$ in the ocean (e.g., Broecker and Peng, 1982). Heavier value of $\delta^{13} \mathrm{C}$ corresponds to higher degree of the uptake and the smaller amount of $\mathrm{\Sigma CO}_{2}$ in the ocean. The inverse correlation between $\delta^{18} \mathrm{O}$ and $\delta^{13} \mathrm{C}$ suggests that glacial stages with low sea level had larger amount of $\mathrm{\Sigma CO}_{2}$, and interglacial stages with high sea level had smaller amount of $\mathrm{\Sigma CO}_{2}$ in the ocean. Two processes have been proposed to explain the inverse relation between $\delta^{18} \mathrm{O}_{\mathrm{B}}$ and $\delta^{13} \mathrm{C}_{\mathrm{B}}$ (Broecker, 1982). One is accumulation of light carbon ${ }^{12} \mathrm{C}$ in the increased and decreased terrestrial biomass associated with humus in interglacial and glacial stages, respectively (Shackleton, 1977), and the other is accumulation and oxidation of light carbon in the "shelf" sediments including fluvial deltaic, lagoonal, alluvial fan, and mangrove swamp, which would respond to glacio-eustatic sea level change in transgressive and regressive stages, respectively (Niitsuma and $\mathrm{Ku}, 1977)$. The responses in the former case are thought of as more reversible and with shorter time constant during glacial-interglacial cycles, but in the latter case completely different processes, deposition and oxidation of "shelf" sediments, relate to the carbon isotopic change. The time constant for the deposition can be thought of as longer than that for oxidation, and the rate of accumulation of light carbon might be nearly constant during a transgression, and not affected by the rate of transgression. However, the rate of oxidation should be followed to the regression, because the exposed organic carbon under the atmosphere might be oxidized suddenly.

The different manner of the isotopic changes $\delta^{18} \mathrm{O}$ and $\delta^{13} \mathrm{C}$ in Site 723 agrees with the "shelf" sediment model, because of the different responses in the transgressive and regressive stages, and the changing rate of $\delta^{13} \mathrm{C}$ is nearly constant during transgressive stages.

The difference in the carbon isotope of benthic and planktonic $\delta^{13} \mathrm{C}_{\mathrm{B}-\mathrm{P}}$ decreases from $-1.6 \%$ to $-0.1 \%$ with depth (Fig. $3)$. The fluctuation of the benthic $\delta^{13} C_{B}$ above Stage 6 is greater than the planktonic $\delta^{13} \mathrm{C}_{\mathrm{P}}$, and the difference $\delta^{13} \mathrm{C}_{\mathrm{B}-\mathrm{P}}$ changes periodically synchronized with the fluctuation of $\delta^{18} \mathrm{O}_{\mathrm{p}}$. The greater differences appeared in the glacial interval, Stage 2,
Stage 6, and Stage 12, and the differences in the interglacial interval, Stage 1 and Stage 5, are similar values to those below Stage 7 (Fig. 4).

Because the carbon isotopic values of benthic $\delta^{13} \mathrm{C}_{\mathrm{B}}$ and planktonic $\delta^{13} C_{\mathrm{P}}$ fluctuated in a parallel manner, the isotopic values represent mainly changes of carbon isotope of the dissolved $\mathrm{\Sigma CO}_{2}$ in the water mass. The fluctuations of difference between benthic and planktonic $\delta^{13} \mathrm{C}_{\mathrm{B}-\mathrm{P}}$ might represent the local changes in the depth profile of the biogenic productivity and/or bottom water chemistry. For example, the heavier planktonic carbon isotopic values $\delta^{13} C_{P}$ appear in the glacial time compared with the benthic values $\delta^{13} \mathrm{C}_{\mathrm{B}}$, and the heavier carbon isotopic values in the surface water indicate higher depletion of light carbon by biological uptake from dissolved $\Sigma \mathrm{CO}_{2}$ in the surface water, derived by upwelling.

\section{RECONSTRUCTION OF UPWELLING HISTORY}

The reconstruction of the upwelling history in the northwestern Indian Ocean was the primary object of the Neogene Package of ODP Leg 117. Site 723 is sitting in the center of the upwelling area and in the central part of the oxygen minimum zone, and also has high resolution data because of the high rate of sedimentation. In the following sections, we will discuss the history of the upwelling by combining isotope data, mentioned above, with other available data.

\section{Available Data for Discussion}

Many kinds of analyses and measurements have been carried out on the sedimentary sequence of the drilled cores from Site 723. In this discussion, we will concentrate mainly on the last $600 \mathrm{ka}$ because of the availability of detailed data sets. The available data and their meaning are listed as follows.

\section{Isotopic data.}

$\delta^{18} \mathrm{O}_{\mathrm{p}}$ : Oxygen isotope of planktonic foraminifer Pulleniatina obliquiloculata; global change of oxygen isotopic composition of seawater.

$\delta^{18} \mathrm{O}_{\mathrm{B}}$ : Oxygen isotope of benthic foraminifer Uvigerina excellens.

$\delta^{18} \mathrm{O}_{\mathrm{B}-\mathrm{p}}$ : Difference between planktonic and benthic oxygen isotope value; bottom temperature at $800 \mathrm{~m}$ depth; indicator of the depth of thermocline.

$\delta^{13} \mathrm{C}_{\mathrm{p}}$ : Carbon isotope of planktonic foraminifer Pulleniatina obliquiloculata.

$\delta^{13} \mathrm{C}_{\mathrm{B}}$ : Carbon isotope of benthic foraminifer Uvigerina excellens; global change of carbon isotopic composition of dissolved $\mathrm{\Sigma CO}_{2}$ in seawater.

$\delta^{13} \mathrm{C}_{\mathrm{B}-\mathrm{P}}$ : Difference between planktonic and benthic carbon isotope value; balance of upwelling-productivity in surface water mass.

\section{Shipboard data, described in Prell, Niitsuma, et al. (1989).}

Sus: Bulk magnetic susceptibility measured with $10 \mathrm{~cm}$ interval; content of terrigenous matter in the sediments.

$\mathrm{CaCO}_{3}$ : Calcium carbonate content measured with $75 \mathrm{~cm}$ interval (two measurements/section); content of calcareous fossils and detrital calcite.

$\mathrm{C}_{\text {org }}$ : Organic carbon content measured with $150 \mathrm{~cm}$ interval (one measurement/section); preserved organic carbon in the sediments.

\section{Shore-based data.}

L: Lightness of sediment color on core photograph measured with video densitometer; the gradations are ranged from 
30 for extreme black part to 220 for extreme white part on the black and white photographs (Niitsuma, this volume).

Sed: Rate of sedimentation based on the oxygen isotope stratigraphy and SPECMAP time table (Imbrie et al., 1984).

\section{Correlation Group}

The available data are cyclic in their values, related to the glacial-interglacial climatic change (Fig. 4). In this discussion, we used oxygen isotope of planktonic $\delta^{18} \mathrm{O}_{\mathrm{P}}$ as an indicator of the glacial-interglacial climatic change and oxygen isotope stratigraphic stages for the description of glacial-interglacial cycle. At first, we divide the available data sets into positive and negative correlation groups with $\delta^{18} \mathrm{O}_{\mathrm{p}}$ which is larger (heavier) value in the glacial stage and smaller (lighter) value in the interglacial stage.

1. Positive correlation group, which has higher or larger values in the glacial stage: $\delta^{18} \mathrm{O}_{\mathrm{B}}$, Sus, L, and Sed.

2. Negative correlation group, which has higher or large values in the interglacial stage: $\delta^{13} \mathrm{C}_{\mathrm{P}}, \delta^{13} \mathrm{C}_{\mathrm{B}}, \delta^{18} \mathrm{O}_{\mathrm{B}-\mathrm{P}}, \mathrm{CaCO}_{3}$, and $\mathrm{C}_{\text {org }}$.

The available data are shown in Figure 4; the vertical axes of all plots are selected as upward for interglacial and downward for glacial conditions.

\section{Significant Correlation}

These data have generally good correlations with each other and show glacial-interglacial cycles clearly, but also show complexity. Further detailed correlations were examined for these data sets with isotopic data and the correlations follow.

\section{Organic carbon content, $\mathrm{C}_{\text {org }}$.}

The range of the organic carbon content is from $1 \%$ to $6 \%$. The content increases in the interglacial stages. The values fluctuated in all stratigraphic intervals since Stage 14, $550 \mathrm{ka}$. The minimum values appear in the glacial stages, Stages $2,4,6,8$, 10 , and 12 . The fluctuation and phases generally match well with $\delta^{18} \mathrm{O}_{\mathrm{P}}$, and better matching is realized with $\delta^{18} \mathrm{O}_{\mathrm{B}-\mathrm{P}}$ for Stages 6-14. The transgressive feature from Stage 2 to 1 and minimum at Stage 2 are rather similar to $\delta^{13} \mathrm{C}_{\mathrm{B}-\mathrm{P} \text {. }}$

\section{Magnetic susceptibility, Sus.}

The range of the magnetic susceptibility is from 10 to $130 \times$ $10^{-6} \mathrm{SI}$. The magnetic susceptibility is high in the glacial stages and low in the interglacial stages, and the fluctuation can be correlated with $\delta^{18} \mathrm{O}_{\mathrm{p}}$. The amplitude of the fluctuation decreases significantly before Stage $8,250 \mathrm{ka}$. The feature of change in the amplitude of fluctuation can be also found in $\delta^{13} \mathrm{C}_{\mathrm{B}-\mathrm{P}}$.

\section{Calcium carbonate content, $\mathrm{CaCO}_{3}$.}

The range of the calcium carbonate content is from $40 \%$ to $70 \%$ The content increases in the interglacial stages and decreases in the glacial stages. The amplitude of the fluctuation decreases significantly before Stage $8,250 \mathrm{ka}$. The change in the amplitude of the fluctuation is similar to that of magnetic susceptibility, Sus, and $\delta^{13} \mathrm{C}_{\mathrm{B}-\mathrm{p}}$. The individual peaks and valleys of the graph are matched better with $\delta^{18} \mathrm{O}_{\mathrm{B}-\mathrm{P}}$, especially transgressive features from Stage 2 to 1 and minimum at Stages 3-4.

\section{Lightness of sediment color, L.}

The range of the lightness of sediment color is from 50 to 110. The color density of cored sediments at Site 723 is the darkest of all Leg 117 sites (Niitsuma, this volume). The pattern of the lightness can be expressed as intercalations of the lighter layers with various higher values in dark background with the lightness of 50. The frequency of the intercalations increases in the glacial stages, Stages 2, 4, 6, 10, and 12; however, the general feature does not appear for Stages 7-9, but the fluctuation of the lightness correlates with $\delta^{18} \mathrm{O}_{\mathrm{B}-\mathrm{P}}$.

\section{Rate of sedimentation, Sed.}

Oxygen isotope stratigraphy gave us an age model by using a standard time table. In the study, SPECMAP time table (Imbrie et al., 1984) is used for the calculation of rate of sedimentation. The calculated rate of sedimentation distinctly changed with the glacial-interglacial cycles. The rate in the glacial stages is significantly high, two to four times that of interglacial stages, especially since Stage 8 . The changes of sedimentation rate correlate with $\delta^{13} C_{B}$ and Sus.

\section{Indicator of Upwelling}

The location of Site 723 is in the central part of present upwelling area, which is related to the transport of the surface seawater by the southwest monsoon in summer. The upwelling brings deep water mass to the sea surface. Because the thermocline is pushed upward by the upwelling, and Site 723 is located around the depth range of thermocline, the bottom temperature at Site 723 should be colder in the season of upwelling (Fig. 2). The sea surface temperature (SST) in summer also becomes significantly cool by the upwelling, $23^{\circ}-24^{\circ} \mathrm{C}$, almost the same as the winter SST in this area (Prell, Niitsuma, et al., 1989; Prell and Streeter, 1982; Prell and Hutson, 1979). The cooling range by upwelling is about $4^{\circ}-6^{\circ} \mathrm{C}$ and the upwelling reduces the seasonal fluctuation of SST.

The measured difference of planktonic and benthic oxygen isotopes $\delta^{18} \mathrm{O}_{\mathrm{B}-\mathrm{P}}$ has an amplitude more than $1 \%$ of which equivalent difference of temperature is $4^{\circ} \mathrm{C}$. The corresponding salinity difference is $4 \%$, which is comparable to the influence of the influx of river water in the Bengal Bay (Duplessy, 1982). The $\delta^{18} \mathrm{O}_{\mathrm{B}-\mathrm{P}}$ fluctuation suggests that the bottom temperature is warmer and/or the isotopic composition of bottom water is affected by fresh water influx in the glacial stage.

The lowering of the sea level in the glacial stage should result in the same sense of change for the $\delta^{18} \mathrm{O}_{\mathrm{B}-\mathrm{P}}$ fluctuation; however, the measured temperature gradient is $0.8^{\circ} \mathrm{C} / 100 \mathrm{~m}$ for the depth range from 330 to $800 \mathrm{~m}$ (Fig. 2), and the contribution of $100 \mathrm{~m}$ sea level change for the oxygen isotope is only $0.2 \%$.

SST for the last glacial maximum, Stage 2, has been estimated as almost the same as at present, because of cancelling of the global cooling by the weakened monsoonal upwelling, based on isotopic and faunal data (Prell and Hutson, 1979). These data suggest that the surface water is not the main cause of the $\delta^{18} \mathrm{O}_{\mathrm{B}-\mathrm{P}}$ fluctuation but rather the bottom water.

The cooler temperature of bottom water in the interglacial stage indicated by the $\delta^{18} \mathrm{O}_{\mathrm{B}-\mathrm{p}}$ fluctuation can be explained as the result of depth changes of the thermocline induced by the upwelling with the southwest monsoon. It means that $\delta^{18} \mathrm{O}_{\mathrm{B}-\mathrm{P}}$ is a good indicator of the upwelling, and the larger differences indicate the stronger upwelling.

The upwelling indicator $\delta^{18} \mathrm{O}_{\text {B-p }}$ is larger in the interglacial stages with large amplitudes of glacial-interglacial cycles for Stages 1-13 (Fig. 3). The amplitude of the fluctuations before Stage 13 decreases and the $\delta^{18} \mathrm{O}_{\text {B-P }}$ is larger, which indicates stronger upwelling. The depth of the Stage 13 coincides with the top depth of the intensive gas expansion after coring.

The upwelling seawater contains nutrients which induce productivity of biomass in the surface water. The high productivity supplies organic carbon into the sediments in the upwelling 
area. The relation between stronger upwelling and high organic carbon is consistent with the correlation of the organic carbon content $\mathrm{C}_{\text {org }}$ and upwelling indicator $\delta^{18} \mathrm{O}_{\mathrm{B}-\mathrm{P}}$, i.e., higher $\mathrm{C}_{\mathrm{org}}$ appears in the interglacial stages with strong upwelling.

The upwelling brings $\Sigma \mathrm{CO}_{2}$ with lighter carbon isotope, and the planktonic and benthic carbon isotope values should be affected by the upwelling. The isotope ratio of the $\Sigma \mathrm{CO}_{2}$ in the surface water mass is controlled by the balance of the upwelling and productivity. The difference of the planktonic and benthic carbon isotope $\delta^{13} \mathrm{C}_{\mathrm{B}-\mathrm{P}}$ is large in the case that the biological uptake exceeds the supply of lighter $\Sigma \mathrm{CO}_{2}$ by upwelling, and is small in the case that upwelling exceeds the biological uptake. Because the measured $\delta^{13} \mathrm{C}_{\mathrm{B}-\mathrm{P}}$ is smaller in the interglacial stages, the supply of lighter $\Sigma \mathrm{CO}_{2}$ is estimated to exceed the biological uptake by the stronger upwelling. The correlation suggests that we can use $\delta^{13} \mathrm{C}_{\mathrm{B}-\mathrm{P}}$ also as an indicator of the upwelling. The glacial-interglacial cycles of the $\delta^{13} \mathrm{C}_{\mathrm{B}-\mathrm{P}}$ were unclear for the interval from Stage 8 to 11. The reduction of the amplitude of fluctuation of $\delta^{13} \mathrm{C}_{\mathrm{B}-\mathrm{P}}$ and its small value indicate that the proportion of the uptake for the biological productivity did not increase in the glacial stages, because of the low organic carbon content $\mathrm{C}_{\text {org. }}$. A similar trend can be found in the calcium carbonate content $\mathrm{CaCO}_{3}$.

\section{Magnetic Susceptibility and Terrigenous Matter}

Oxygen isotope curve has positive correlation with susceptibility curve; the interglacial stage has low susceptibility values, and the glacial stage has high susceptibility values. The susceptibility is controlled by the total amount of magnetic minerals in the sample, and the magnetic minerals are thought of as terrigenous in origin. The fluctuation of the magnetic susceptibility indicates that the supply of magnetic minerals decreased in the interglacial stage. Because the strength of the monsoon is estimated to increase in the interglacial stage, based on both of the upwelling indicators $\delta^{18} \mathrm{O}_{\mathrm{B}-\mathrm{P}}$ and $\delta^{13} \mathrm{C}_{\mathrm{B}-\mathrm{P}}$, the supply of terrigenous matter decreased in the interglacial stage with strong southwest monsoon.

The southwest monsoon is the most favorable carrier for the eolian transportation of terrigenous matter from the desert in the Arabian Peninsula and North Africa to the Oman Margin. If the main part of terrigenous matter in the Oman Margin sediments was transported as eolian grains by the southwest monsoon, the flux of the terrigenous matter should increase in the interglacial stage with strong monsoon. However, the fluctuation of the susceptibility indicates that the content of the terrigenous matter decreased in the interglacial stage. The reverse relation can be explained only by the dilution of terrigenous matter with the biogenic products relating to the strong upwelling in the interglacial. In such case, the rate of sedimentation during interglacial stages should be higher than in the glacial stage; however, the rate of sedimentation in the interglacial stage is significantly lower than in glacial stage, as mentioned above. These sets of data suggest that the main part of the terrigenous matter in the Oman Margin sediments were not transported as eolian particles by southwest monsoon.

Another way to explain the fluctuation of the terrigenous matter is by the fluvial transportation. The fluvial transportation is capable of carrying the large amount of terrigenous matter from the land area to the continental margin, and the amount might be sensitive to the climatic condition of humid or arid. The lower sea level in the glacial stage should be also effective in increasing the supply of the terrigenous matter to the sedimentary basin on the continental slope.

The distinct contrast in the strength of magnetic susceptibility between glacial and interglacial stages started from oxygen Stage 7 or 8 .

\section{Monsoon and Aridity of the Arabian and North African Desert}

The magnetic susceptibility data and isotopic upwelling indicators clearly suggest the increase in the supply of the terrigenous matter in the glacial stage. The layer-by-layer correlation indicates that the increase of the sediment supply is not a local phenomenon at Site $\mathbf{7 2 3}$ but also all over the Oman Margin, and that the absolute flux of the sediments increased in the glacial stage; the sediments have various facies which indicate the changeable circumstance of the sediment supply (Niitsuma, this volume). These data suggest that the large amount of terrigenous matter in the glacial stage is transported from the land area by the drainage systems.

Meteorological studies show that the southwest monsoon is induced by the strong ascending current on the Himalaya-Tibet high-mountainous area in summer, and the counter-current of the southwest monsoon develops the Tropical Easterly Jet from southeast Asia to north Africa (Hahn and Manabe, 1975; Barnett et al., 1989). The Easterly Jet is directly related to the high precipitation in southeast Asia and arid climate in north Africa and the Arabian Peninsula. Because the arid climate of the Arabian Peninsula is maintained by the strong monsoon today, the glacial stage with weak monsoon and weak upwelling had a more humid climate in the Arabian Peninsula. The relation agrees better with the fluvial origin of the sediments in the glacial stages.

Although Kutzbach (1986) mentioned that lake levels in Africa were low in the last glacial, based on the data of Butzer et al. (1972) and Street and Grove (1979), humid conditions in the last glacial stage have been reported from the lake sediments of the Dead Sea and Zeribar in the Middle East and Lake Mundafan in the Arabian Peninsula (Street and Grove, 1979). Lake deposition of the Lake Mundafan in the central part of the Arabian Peninsula were classified into Series A, B, and C based on their radiocarbon date and altitude, and their dates are 36-17 $\mathrm{ka}, 9-6 \mathrm{ka}$, and 15-11 ka, respectively. Generally, beds of Series A occupy high parts of the basin and beds of Series B and C occupy low parts (McClure, 1976). The humid records during glacial stage support the fluvial origin of the sediment supply in glacial stages.

\section{History of the Upwelling}

The detailed history of the upwelling can be reconstructed with the available data sets since Stage $15,600 \mathrm{ka}$. The most useful upwelling indicator $\delta^{18} \mathrm{O}_{\mathrm{B}-\mathrm{p}}$ related to the depth of thermocline indicates that the upwelling in the Oman Margin developed more strongly in the interglacial stages. The present glacial-interglacial cycle can be traced back to Stage $8,250 \mathrm{ka}$, with the cyclic changes of the other upwelling indicator $\delta^{13} \mathrm{C}_{\mathrm{B}-\mathrm{P}}$. The cycle upheld the arid-humid cycle of the Arabian and North African Desert, and large amounts of terrigenous input were supplied in the glacial stage.

For the interval from Stage 8 to $12,250-450 \mathrm{ka}$, the upwelling indicator $\delta^{18} \mathrm{O}_{\mathrm{B}-\mathrm{P}}$ was still signifying the distinct glacial-interglacial cycle, but the other upwelling indicator $\delta^{13} \mathrm{C}_{\mathrm{B}-\mathrm{P}}$ did not show such distinct cyclicity.

For Stages 12-15, 450-600 ka, the glacial-interglacial cycles can be traced back, however, the value of $\delta^{18} \mathrm{O}_{\mathrm{B}-\mathrm{P}}$ remained strong in the upwelling side, and the sediments contain high concentration of gas. $\delta^{13} C_{\mathrm{B}-\mathrm{P}}$ recovered the glacial-interglacial cyclicity as in present cycles. This interval corresponds to the increasing stage of the global ice sheet volume from the pre-glacial cycles with small amplitude of oxygen isotope fluctuation to present glacial-interglacial cycles. The situations of upwelling can be estimated as strong as in the interglacial stage of the 
present cycles, with slightly weak upwelling in the glacial stage. The cyclic input of the fluvial sediments to present cycles cannot be estimated.

Before Stage 15, $600 \mathrm{ka}$, the available data are limited because of the incomplete core recovery of and gas expansion of the cored sediments. Based on the isotopic results, the situations of upwelling and climate for Stage 12 to 15 can be traced back to the late Pliocene. The difference of planktonic and benthic oxygen isotope $\delta^{18} \mathrm{O}_{\mathrm{B}-\mathrm{P}}$ increases with depth (Fig. 3). Because the difference $\delta^{18} \mathrm{O}_{\mathrm{B}-\mathrm{P}}$ is good indicator of strength of upwelling, as mentioned above, the much stronger upwelling can be estimated in the late Pliocene to early Pleistocene time. The difference of carbon isotope $\delta^{13} \mathrm{C}_{\mathrm{B}-\mathrm{P}}$ also gradually lessens, which indicated the stronger upwelling. The strong upwelling indicated by isotopic results is consistent with the high organic carbon content (Fig. 3), existence of thin laminated sedimentary structures which are characteristic of the anoxic condition under the high productive area of upwelling, and high content of gas in the sediments, which causes poor recovery of cored sediments and disturbance by gas expansion (Prell, Niitsuma, et al., 1989).

\section{SUMMARY AND CONCLUSION}

This paper mentions the results of isotopic measurements on the sedimentary sequence of Site 723, and discusses the history of the upwelling in the Oman Margin and paleoclimatology, related to the southwest monsoon in the northwestern Indian Ocean.

Site 723 is located in the center of mid-slope basin of the Oman margin, and high resolution data are available because of the high rate of sedimentation. The water depth of $808 \mathrm{~m}$ is situated on the center of the oxygen minimum zone developing under the upwelling area and on the middle part of the thermocline.

Oxygen isotope curves of planktonic $\delta^{18} \mathrm{O}_{\mathrm{P}}$ and benthic $\delta^{18} \mathrm{O}_{\mathrm{B}}$ can be correlated well with standard curves and can be traced back continuously to Stage 23 with high resolution measurements. The tentative correlation has been tried to Stage 53. The amplitudes of the fluctuations of the benthic $\delta^{18} \mathrm{O}_{\mathrm{B}}$ curve are significantly small, especially older than Stage 16, compared with planktonic $\delta^{18} \mathrm{O}_{\mathrm{p}}$.

Significant delays of benthic oxygen isotopes $\delta^{18} \mathrm{O}_{\mathrm{B}}$ appear in the transgressive stage from the planktonic $\delta^{18} \mathrm{O}_{\mathrm{p}}$. The delays are counted for depth $50-80 \mathrm{~cm}$ whose corresponding duration is calculated to be $2500-4000 \mathrm{yr}$.

Generally, carbon isotopes of benthic $\delta^{13} \mathrm{C}_{\mathrm{B}}$ and planktonic $\delta^{13} \mathrm{C}_{\mathrm{P}}$ have inverse correlation with oxygen isotope values $\delta^{18} \mathrm{O}_{\mathrm{B}}$ and $\delta^{18} \mathrm{O}_{\mathrm{P}}$, however, the changes of $\delta^{13} \mathrm{C}$ are more gradual than those of $\delta^{18} \mathrm{O}$ during transgressive stages in spite of the synchronized changes of $\delta^{13} \mathrm{C}$ with those of $\delta^{18} \mathrm{O}$ during regressive stages, which is agreeable with the "shelf" model for global carbon isotope fluctuation in the deep water mass.

The difference of oxygen isotope $\delta^{18} \mathrm{O}_{\text {B-p }}$ indicates that the bottom temperature was warmer in the glacial stage than in the interglacial stage. The relation can be explained by the pushedup thermocline during the strong upwelling stage in the interglacial stages. It means that $\delta^{18} \mathrm{O}_{\mathrm{B}-\mathrm{P}}$ is a good indicator of the upwelling, and the larger differences indicate the stronger upwelling in the interglacial stages.

The difference of carbon isotope $\delta^{13} \mathrm{C}_{\mathrm{B}-\mathrm{P}}$ also can be used as an indicator of the upwelling, because the smaller values of $\delta^{13} \mathrm{C}_{\mathrm{B}-\mathrm{P}}$ appear in the interglacial stages with stronger upwelling estimated by $\delta^{18} \mathrm{O}_{\mathrm{B}-\mathrm{p}}$, and the small values mean that upwelling $\Sigma \mathrm{CO}_{2}$ exceeds the biological uptake.

The organic carbon content $\mathrm{C}_{\text {org }}$ is correlated with isotopic upwelling indicators $\delta^{18} \mathrm{O}_{\mathrm{B}-\mathrm{P}}$ and $\delta^{13} \mathrm{C}_{\mathrm{B}-\mathrm{p}}$, i.e., higher $\mathrm{C}_{\mathrm{org}}$ appears in the interglacial stages with strong upwelling.
The calculated rate of sedimentation based on oxygen isotope stratigraphy in glacial stages is significantly high, two to four times that of interglacial stages. The isotopic upwelling indicators and the layer-by-layer correlation and magnetic susceptibility data show that the absolute flux of terrigenous matter and variability of lithofacies increased in the glacial stage. The terrigenous matter in the glacial stage is estimated to be transported from humid Arabian Peninsula under weak condition of Tropical Easterly Jet.

The present glacial-interglacial cycle with upwelling fluctuation and arid-humid alternation can be traced back to Stage 8 , $250 \mathrm{ka}$. For the interval from Stage 8 to $12,250-450 \mathrm{ka}$, the upwelling indicator $\delta^{18} \mathrm{O}_{\mathrm{B}-\mathrm{P}}$ still observed the distinct glacial-interglacial cycle, but the other upwelling indicator $\delta^{13} \mathrm{C}_{\mathrm{B}-\mathrm{P}}$ did not show such distinct cyclicity.

For Stages 12-15, 450-600 ka, the upwelling can be estimated as strong as in the interglacial stage of the present cycles, with slightly weak upwelling in the glacial stage. This upwelling and climate can be traced back to the late Pliocene. The strongest upwelling can be estimated in the late Pliocene to early Pleistocene by the isotopic indicators and the high organic carbon content.

\section{ACKNOWLEDGMENTS}

We would like to thank Warren Prell of Brown University, William Curry of Woods Hole Oceanographic Institution, and Alan Mix for critical review, comments, and discussions; John Imbrie, Dave Murray, and Steven Clemens of Brown University, Susumu Honjo of Woods Hole Oceanographic Institution, Akira Hayashida of Doshisha University, Toshiaki Takayama of Kanazawa University, Stacia Spaulding of University of Nebraska, Dick Kroon of Vrije University, Hideki Wada of Shizuoka University, Asahiko Taira of University of Tokyo, Tetsuzo Yasunari of Tsukuba University, and Masato Shinoda of Tokyo Metropolitan University for valuable comments and discussions; and Norman Stewart of ODP for editorial help and improvement of English.

\section{REFERENCES}

Barnett, T. P., Dumenil, L., Schlese, U., Roeckner, E., and Latif, M. 1989. The effect of Eurasian snow cover on regional and global climate variations. J. Atmos. Sci., 46:661-668.

Broecker, W. S., 1982. Ocean chemistry during glacial time. Geochim. Cosmochim. Acta, 46:1689-1705.

Broecker, W. S., and Peng, T.-H., 1982. Tracers in the Sea: Palisades, NY (Eldigio Press).

Broecker, W. S., and vanDonk, J., 1970. Insolation changes, ice volumes, and the $\mathrm{O}^{18}$ record in deep-sea cores. Rev. Geophys. Space Phys., 8:169-198.

Butzer, K. W., Isaac, G. L., Richardson, J. A., and Washbourn-Kamau, C., 1972. Radiocarbon dating of East African lake levels. Science, 175:1069-1076.

Craig, H., 1957. Isotopic standards for carbon and oxygen correction factors for mass spectrometric analysis of carbon dioxide. Geochim. Cosmochim. Acta, 12:133-149.

Duplessy, J. C., 1982. Glacial to interglacial contrasts in the northern Indian Ocean. Nature, 295:494-498.

Hahn, D. G., and Manabe, S., 1975. The role of mountains in the South Asian monsoon circulation. J. Atmos. Sci., 32:1515-1541.

Imbrie, J., Hays, J. D., Martinson, D. G., McIntyre, A., Mix, A. C., Morley, J. J., Pisias, N. G., Prell, W. L., and Shackleton, N. J., 1984. The orbital theory of Pleistocene climate: support from a revised chronology of the marine $\delta^{18} \mathrm{O}$ record. In Berger, A., Imbrie, J., Hays, J., Kukla, G., and Saltzman, B. (Eds.), Milankovitch and Climate (Pt. 1): Dordrecht (D. Reidel), 269-305.

Kahn, M. I., 1979. Non-equilibrium oxygen and carbon isotopic fractionation in tests of living planktonic foraminifera. Oceanol. Acta, 2:195-208. 
Kutzbach, J. E., 1986. The changing pulse of the Monsoon. In Fein, J. S., and Stephens, P. L. (Eds.), Monsoons: New York (Wiley), 247268.

McClure, H. A., 1976. Radiocarbon chronology of the Quaternary lakes in the Arabian Desert. Nature, 263:755-756.

Niitsuma, N., and Ku, T. L., 1977. What happened during geomagnetic reversals? Kagaku, 47:671-678.

Prell, W. L., and Hutson, W. H., 1979. Zonal temperature-anomaly maps of Indian Ocean surface waters: modern and ice-age patterns. Science, 206:454-456.

Prell, W. L., Imbrie, J., Martinson, D. G., Morley, J. J., Pisias, N. G., Shackleton, N. J., and Streeter, H. F., 1986. Graphic correlation of oxygen isotope stratigraphy application to the late Quaternary. Paleoceanography, 1:137-162.

Prell, W. L., Niitsuma, N., et al., 1989. Proc. ODP, Init. Repts., 117: College Station, TX (Ocean Drilling Program).

Prell, W. L., and Streeter, H. F., 1982. Temporal and spatial patterns of monsoonal upwelling along Arabia: a modern analogue for the in- terpretation of Quaternary SST anomalies. J. Mar. Res., 40:143155.

Shackleton, N. J., 1977. Tropical rainforest history and the equatorial Pacific carbonate dissolution cycles. In Anderson, N. R., and Malahoff, A. (Eds.), The Fate of Fossil Fuel $\mathrm{CO}_{2}$ in the Oceans: New York (Plenum), 401-428.

Street, F. A., and Grove, A. T., 1979. Global maps of lake-level fluctuations since 30,000 yr B.P. Quat. Res., 12:83-118.

Wada, H., Fujii, N., and Niitsuma, N., 1984. Analytical method of stable isotope for ultra-small amounts of carbon dioxide with MAT 250 mass-spectrometer. Geosci. Repts. Shizuoka Univ., 10:103-112.

Williams, D. F., Thunnell, R. C., Tappa, E., Rio, D., and Raffi, I., 1988. Chronology of the Pleistocene oxygen-isotope record: $0-1.88$ m.y.B.P. Palaeogeogr., Palaeoclimatol., Palaeoecol., 64:221-240.

Date of initial receipt: 3 October 1989

Date of acceptance: 25 July 1990

Ms 117B-168 